\title{
Heterogeneity and diversity of striatal GABAergic interneurons
}

\author{
James M. Tepper*, Fatuel Tecuapetla ${ }^{\dagger}$, Tibor Koós* and Osvaldo Ibáñez-Sandoval
}

Center for Molecular and Behavioral Neuroscience, Rutgers University, Newark, NJ, USA

Edited by:

Jose L. Lanciego, University of

Navarra, Spain

Reviewed by:

Enrico Bracci, University of

Manchester, UK

Anatol Kreitzer, University of California

San Francisco, USA

${ }^{*}$ Correspondence:

James M. Tepper and Tibor Koós,

Center for Molecular and Behavioral

Neuroscience, Rutgers University, 197

University Avenue, Newark, NJ 07102,

USA.

e-mail: jtepper@andromeda.rutgers.

edu; tibkoos@yahoo.com

\section{tPresent address:}

Fatuel Tecuapetla, Neurobiology of Action, Instituto Gulbenkian de Ciencia, Rua da Quinta Grande, 6, 2780-156 Oeiras, Portugal.
The canonical view of striatal GABAergic interneurons has evolved over several decades of neuroanatomical/neurochemical and electrophysiological studies. From the anatomical studies, three distinct GABAergic interneuronal subtypes are generally recognized. The best-studied subtype expresses the calcium-binding protein, parvalbumin. The second best known interneuron type expresses a number of neuropeptides and enzymes, including neuropeptide $Y$, somatostatin, and nitric oxide synthase. The last GABAergic interneuron subtype expresses the calcium binding protein, calretinin. There is no overlap or co-localization of these three different sets of markers. The parvalbumin-immunoreactive GABAergic interneurons have been recorded in vitro and shown to exhibit a fast-spiking phenotype characterized by short duration action potentials with large and rapid spike AHPs. They often fire in a stuttering pattern of high frequency firing interrupted by periods of silence. They are capable of sustained firing rates of over $200 \mathrm{~Hz}$. The NPY/SOM/NOS interneurons have been identified as PLTS cells, exhibiting very high input resistances, low threshold spike and prolonged plateau potentials in response to intracellular depolarization or excitatory synaptic stimulation. Thus far, no recordings from identified CR interneurons have been obtained. Recent advances in technological approaches, most notably the generation of several BAC transgenic mouse strains which express a fluorescent marker, enhanced green fluorescent protein, specifically and selectively only in neurons of a certain genetic makeup (e.g., parvalbumin-, neuropeptide Y-, or tyrosine hydroxylase-expressing neurons etc.) have led to the ability of electrophysiologists to visualize and patch specific neuron types in brain slices with epifluorescence illumination. This has led to a rapid expansion of the number of neurochemically and/or electrophysiologically identified interneuronal cell types in the striatum and elsewhere. This article will review the anatomy, neurochemistry, electrophysiology, synaptic connections, and function of the three "classic" striatal GABAergic interneurons as well as more recent data derived from in vitro recordings from BAC transgenic mice as well as recent in vivo data.

Keywords: neostriatum, interneuron, GABAergic, tyrosine hydroxylase, EGFP, NPY, SOM, NOS

\section{INTRODUCTION}

It was recognized from the earliest neurocytological studies that the neostriatum comprised a large number of small to medium sized neurons (less than $20 \mu \mathrm{m}$ in diameter) of varying morphology and a small number of large neurons (Mehler, 1981). Estimates of the ratio of small-medium to large cells varied widely in these reports, from 20:1 up to 270:1 due to the lack of application of rigorous quantitative techniques (Parent, 1986). Although the large neurons were first identified by Kölliker (1896) as giant interneurons, Ramon y Cajal (1911), in a rare error, identified them as striatal projection neurons (SPNs), a claim reiterated by Vogt and Vogt (1920), thus presenting a picture of the striatum as a nucleus comprised primarily of interneurons with a very small number of projection neurons (Zhou et al., 2002). This resulted in decades of controversy and confusion about the functional identities of the large and the small striatal cells, that was not resolved conclusively until retrograde labeling from substantia nigra and globus pallidus unambiguously identified the medium sized spiny neurons as the SPN and the giant aspiny neurons as interneurons (Grofova, 1979) that were later shown to be uniformly cholinergic (Kimura et al., 1980).

Striatal projection neurons are now recognized to make up approximately $95 \%$ of all the neurons in the rodent striatum (Gerfen and Wilson, 1996; the proportion is significantly lower in higher vertebrates, especially in primates, Graveland and DiFiglia, 1985), and the cholinergic interneurons make up only $0.5-1 \%$ of the neurons. The remaining neurons, thus comprising approximately $3-4 \%$ of the total number of neurons in the rodent striatum, are made up of several different subtypes of aspiny GABAergic interneurons.

Striatal GABAergic interneurons were first identified as such by their avid uptake of ${ }^{3} \mathrm{H}$-GABA combined with Golgi staining (Bolam et al., 1983). Medium-sized aspiny striatal neurons with varicose dendrites and indented nuclear envelopes accumulated ${ }^{3} \mathrm{H}$-GABA at a rate almost one order of magnitude greater that of the spiny projection neurons. Subsequent studies revealed that a population of aspiny interneurons with similar or identical characteristics also 
exhibited significantly stronger glutamate decarboxylase (GAD) activity than spiny projection neurons (Bolam et al., 1985; Cowan et al., 1990; Kita, 1993; Kubota et al., 1993).

By the mid 1990s, there were reliable reports of three distinct subtypes of medium sized striatal GABAergic interneurons that could be distinguished in striatum of mammalian species on the basis of their expression of the calcium binding proteins parvalbumin (PV) or calretinin (CR), or their expression of the neuropeptides somatostatin (SOM), or neuropeptide Y (NPY) or nitric oxide synthase (NOS) (Vincent and Johansson, 1983; Vincent et al., 1983; Chesselet and Graybiel, 1986; Cowan et al., 1990; Kita et al., 1990; Bennett and Bolam, 1993; Kubota and Kawaguchi, 1993, 1994, 1995; Kubota et al., 1993). Each of these exists in very low abundance compared to the SPNs. In the case of $\mathrm{PV}^{+}$interneurons, $0.7 \%, \mathrm{CR}^{+}$interneurons, $0.5 \%$ and $\mathrm{SOM} / \mathrm{NOS} / \mathrm{NPY}^{+}$interneurons $0.6 \%$, as determined by unbiased stereological cell counts of immunostained rat striatum (Luk and Sadikot, 2001; Rymar et al., 2004). Of course, these numbers should be regarded as minimum estimates of the numbers of GABAergic interneurons, since immunostaining is of relatively low and variable sensitivity compared to other techniques for neurochemical phenotyping, so what this really means is that at least $1.8 \%$ of striatal neurons consist of GABAergic interneurons, and the proportion is almost certainly higher than that.

These reports were followed by groundbreaking in vitro electrophysiological studies by Yasuo Kawaguchi and colleagues that showed that the PV-immunoreactive $\left(\mathrm{PV}^{+}\right)$and SOM-NPY-NOS ${ }^{+}$ neurons also exhibited distinct somatodendritic and axonal morphologies, as well as readily distinguishable electrophysiological properties (Kawaguchi, 1993, 1997; Kubota et al., 1993; Kawaguchi et al., 1995). The first part of this article will concentrate on these earlier studies, reviewing the original core data on the "classic" striatal GABAergic interneurons, as well as updating them with the most recent findings on synaptic connectivity and pharmacology.

More recently, the widespread availability of several strains of transgenic mice engineered to express enhanced green fluorescent protein (EGFP) under the control of endogenous transcriptional regulatory sequences (Gong et al., 2003) has provided researchers with additional powerful tools with which to target specific neuronal subtypes for visually guided recording, thereby simplifying the electrophysiological characterization and biocytin labeling of different striatal GABAergic interneurons. These in turn have resulted in an expansion of the number of distinct subtypes of striatal GABAergic interneurons that have been electrophysiologically and morphologically characterized. These data will be reviewed in the next part of this article.

We will conclude with a discussion of the changing concepts of striatal organization and the functional diversity and potential roles of striatal GABAergic interneurons.

\section{$\mathrm{PV}^{+}$INTERNEURONS NEUROCYTOLOGY}

Parvalbumin-immunoreactive striatal neurons were first reported by Gerfen et al. (1985) who showed the existence of medium-sized, aspiny interneurons that were distinct from previously described cholinergic and $\mathrm{SOM}^{+}$neurons, and which were more abundant laterally than medially. Subsequent studies revealed that these neurons were strongly immunoreactive for GAD (Kita et al., 1990), particularly the $\mathrm{GAD}_{67}$ isoform (Lenz et al., 1994) and were present in both patch and matrix compartments with dendrites that freely crossed patch-matrix boundaries (Cowan et al., 1990) and thus were almost certainly the striatal GABAergic interneurons that were originally identified on the basis of intense ${ }^{3} \mathrm{H}$-GABA uptake (Bolam et al., 1983).

Most $\mathrm{PV}^{+}$striatal interneurons are categorized as mediumsized (Kita et al., 1990; Kawaguchi, 1993; Koós and Tepper, 1999), although a rare variant has been described that can be as large as the giant cholinergic interneuron (Bennett and Bolam, 1994a). In a sample of intracellularly labeled $\mathrm{PV}^{+}$interneurons Kawaguchi (1993) described two distinct morphologies, one characterized by a medium size soma and more compact axonal and dendritic fields, and another exhibiting larger somatic diameters as well as axonal and dendritic fields. These observations suggest that $\mathrm{PV}^{+}$ interneurons may not represent a single homogenous cell type. $\mathrm{PV}^{+}$interneurons are not distributed homogeneously throughout striatum; rather they obey a ventral to dorsal, medial to lateral, and caudal to rostral gradient of increasing density (Gerfen et al., 1985; Kita et al., 1990; Mura et al., 2000; Wu and Parent, 2000). PV $^{+}$ interneurons emit five to eight aspiny dendrites that range from almost smooth to extremely varicose (Cowan et al., 1990; Kita et al., 1990; Kawaguchi, 1993; Kita, 1993). Most dendrites branch within a few $10 \mathrm{~s}$ of $\mu \mathrm{m}$ from the soma, and then only sparingly, with dendrites greater than third or fourth order rare. The entire dendritic field is compact and roughly spherical, extending 200-300 $\mu \mathrm{m}$ in diameter centered around the soma. A relatively small fraction of $\mathrm{PV}^{+}$neurons display more extended dendritic fields (Kawaguchi, 1993).

The axon is highly branched, extends through and beyond the dendritic tree of the parent cell and is one of the densest axonal arborizations of any striatal neuron. The axonal field is also roughly spherical or ovoid and extends about 1.5-2 times the diameter of the dendritic field with its maximal density roughly coextensive with the dendritic arborization (Kawaguchi, 1993; Koós and Tepper, 1999; Tepper and Bolam, 2004).

In EM studies the nuclear envelope of $\mathrm{PV}^{+}$neurons is deeply invaginated (Kita et al., 1990), consistent with that of the aspiny GABAergic interneurons described previously (Bolam et al., 1983; Takagi et al., 1984a). In addition, striatal $\mathrm{PV}^{+}$dendrites were observed to form dendro-dendritic gap junctions with other $\mathrm{PV}^{+}$ dendrites (Kita et al., 1990; Kita, 1993). $\mathrm{PV}^{+}$interneurons are the only striatal neuron of any type where gap junctions have been directly observed morphologically.

\section{INTRINSIC ELECTROPHYSIOLOGICAL PROPERTIES}

Striatal $\mathrm{PV}^{+}$interneurons exhibit a distinctive electrophysiological profile that enables them to be identified strictly on that basis of intracellular or whole cell recordings in vitro or in vivo. The electrophysiological characteristics of striatal $\mathrm{PV}^{+}$interneurons were first identified in slices from juvenile rats by Kawaguchi and colleagues (Kawaguchi, 1993; Kawaguchi et al., 1995), and these observations have subsequently been replicated in slices from mature rats and mice (Koós and Tepper, 1999, 2002; Bracci et al., 2002, 2003; Centonze et al., 2003; Plotkin et al., 2005; Taverna et al., 2007; Ibáñez-Sandoval et al., 2010). 
All striatal $\mathrm{PV}^{+}$interneurons are classified electrophysiologically as fast-spiking interneurons (FSI) and in some instances can fire at frequencies over $400 \mathrm{~Hz}$ (Figure 1C) in response to strong depolarizing current injections with little spike frequency adaptation (Figures 1A,C). $\mathrm{PV}^{+}$FSI are strongly hyperpolarized in vitro and do not exhibit spontaneous activity. They exhibit a low input resistance similar to that of SPNs (50-150 M $\Omega$ ), but significantly less inward rectification. Action potentials evoked by depolarizing current injection are of short duration $(<0.5 \mathrm{~ms})$ and exhibit deep, rapid onset and short duration spike after hyperpolarizations. Similar characteristics are exhibited by FSI in cortex and hippocampus that are also $\mathrm{PV}^{+}$(Freund and Buzsáki, 1996; Galarreta and Hestrin, 1999, 2001, 2002) and are likely due, at least in part to the expression of $\mathrm{Kv} 3.1$, a high threshold, rapidly activating and slowly inactivating voltage-gated $\mathrm{K}^{+}$channel that is expressed selectively in $\mathrm{PV}^{+} \mathrm{FS}$ interneurons (Lenz et al., 1994; Rudy and McBain, 2001).

Characteristically, unlike most neurons, the intrinsic ionic mechanisms of striatal $\mathrm{PV}^{+}$interneurons cannot sustain repetitive firing at arbitrarily low frequencies. As shown in Figure 1, increasing the amplitude of current injection steps finally depolarizes the neuron to threshold (about $-50 \mathrm{mV}$ ), eliciting a single, delayed spike. However, further small increments of injected current do not elicit gradually increasing rates of firing, instead, a tiny increment in stimulus strength of $10 \mathrm{pA}$ above threshold results in firing at the neuron's minimal sustainable frequency typically exceeding $20 \mathrm{~Hz}$ (Figure 1).
Among neostriatal interneurons defined as FSIs based on these electrophysiological criteria two subtypes may be distinguished based on the temporal structure of action potential trains elicited with current injection. One of these is characterized by continuous firing that is maintained for the duration of the current injection except for very high current amplitudes where the action potential train is sometimes terminated earlier, presumably due to $\mathrm{Na}^{+}$channel inactivation. The other type of FSIs exhibit a "stuttering" response consisting of brief trains of action potentials separated by silent periods of variable duration during which subthreshold membrane potential oscillations are observed (Kawaguchi, 1993; Koós and Tepper, 1999; Bracci et al., 2003). It remains unclear if these two firing patterns reflect different states of the same neurons or are indicators of genuinely distinct neuronal phenotypes. In one study using BAC-PV-EGFP transgenic mice that enabled immunocytochemically verifiable targeting of $\mathrm{PV}^{+}$interneurons Freiman et al. (2006) observed only the continuous firing pattern in $\mathrm{EGFP}^{+}$FSIs. In contrast, FSIs exhibiting stuttering responses have been shown to stain for PV using immunocytochemistry in the rat neostriatum (Kawaguchi, 1993) and nucleus accumbens (Taverna et al., 2007). In addition, both firing types have been reported in both rats (Bracci et al., 2002, 2003; Plotkin et al., 2005; Taverna et al., 2007) and mice (Centonze et al., 2003; Gittis et al., 2010) among electrophysiologically defined FSIs, although the stuttering phenotype appears to be less frequent in mice than in rats (Tecuapetla et al., unpublished). Since developmental effects cannot account for these findings (Plotkin et al., 2005) the data together suggest

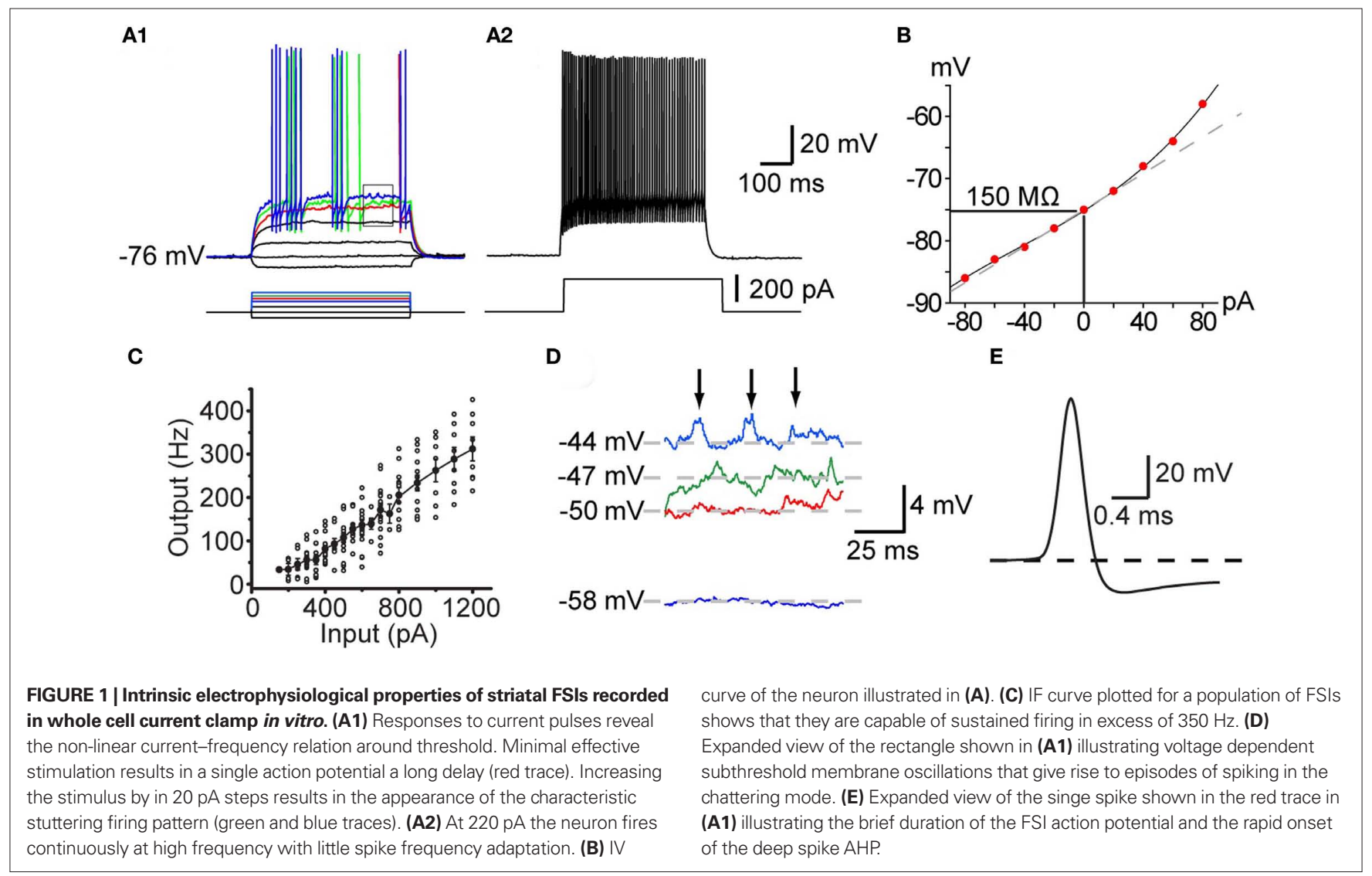


either a species and cell type specific difference in the expression of PV, so that in mice PV would be present only in continuous firing but not in stuttering FSIs; while in the rat, PV would be expressed in FSIs exhibiting either characteristics, or alternatively, stuttering neurons may be $\mathrm{PV}^{+}$in the mouse but much less frequent of much less frequently encountered in slice experiments than in rats. These interpretations should be considered tentative however, until the findings of Freiman et al. (2006) are confirmed with a more systematic characterization of a larger sample of neurons.

Another characteristic feature of these neurons is the presence of large amplitude (2-3 mV) voltage dependent membrane oscillations at depolarized subthreshold membrane potentials (Koós and Tepper, 1999; Bracci et al., 2003), which is particularly prominent in stuttering neurons during the periods between action potentials bursts. In stuttering neurons the oscillations appear to trigger the firing of bursts by bringing the membrane potential to threshold. The oscillations and the intermittent firing pattern are $\mathrm{Ca}^{2+}$ or SK channel independent, but are completely eliminated by TTX, suggesting that they are due to an interaction between voltage-gated $\mathrm{K}^{+}$conductances and a persistent or possibly the inactivating sodium conductance responsible for spike generation (Bracci et al., 2003, Koós and Tepper, unpublished).

An additional characteristic of striatal $\mathrm{PV}^{+}$interneurons is that they are interconnected by electrotonic synapses (Koós and Tepper, 1999), as are PV ${ }^{+}$FSI in cortex and hippocampus (Galarreta and Hestrin, 2001). Although the coupling ratio is usually not strong enough to evoke spiking, it is powerful enough to allow evoked spikes in electrotonically coupled FSI to fire near synchronously (Tepper, 2010). Although it has been proposed that this would allow syncytial activation of groups of FSI firing in near synchrony (e.g., Kita et al., 1990; Koós and Tepper, 1999), recent in vivo recordings from presumed FSI showed that striatal FSI activity is largely uncoordinated, at least during the cue and reward phases of a conditioned maze task (Berke, 2008).

\section{AFFERENT CONNECTIVITY}

Both symmetric and asymmetric synapses are seen to contact $\mathrm{PV}^{+}$ dendrites and somata. Somatic inputs of both types are relatively sparse, but the dendrites are heavily innervated. Nearly two-thirds of the afferents form asymmetric synapses (Kita et al., 1990), originating mostly from cortex, with rather little thalamic innervation (Kita, 1993). Single cortical axons make multiple contacts with $\mathrm{PV}^{+}$interneurons (Ramanathan et al., 2002), which may account, in part, for the greater responsivity of $\mathrm{PV}^{+}$interneurons to cortical stimulation (Parthasarathy and Graybiel, 1997) compared to SPNs (Mallet et al., 2005). The symmetric synapses arise from both extrinsic and intrinsic GABAergic and dopaminergic inputs (Kubota et al., 1987; Bevan et al., 1998).

Choline acetyltransferase immunoreactive boutons have been seen in contact with $\mathrm{PV}^{+}$somata and dendrites in striatum (Chang and Kita, 1992), and striatal FSI are powerfully excited by stimulation of non-desensitizing nicotinic receptors in vitro (Koós and Tepper, 2002). However, at present, there have been no demonstrations of EPSP/Cs ${ }^{+}$originating from stimulation of cholinergic interneurons.

Dopaminergic inputs to presumed $\mathrm{PV}^{+}$interneurons have also been observed ultrastructurally (Kubota et al., 1987), as have GABAergic inputs originating from the GP (Bevan et al., 1998).
The pallidostriatal inputs are largely selective for FSIs (Bevan et al., 1998) and in vivo, increased firing of FSI during choice selection in a simple discrimination task coincide with a decrease in firing of GP neurons (Gage et al., 2010).

\section{EFFERENT CONNECTIVITY}

Action potentials in striatal FSI evoke large amplitude IPSPs in SPNs in organotypic cell culture (Plenz and Kitai, 1998) and in acute striatal slices (Koós and Tepper, 1999, 2002). Synapses between striatal FSIs and SPNs are largely proximal (Kita et al., 1990; Kita, 1993; Bennett and Bolam, 1994b) and paired recordings show that they exhibit extremely low failure rates $(<1 \%)$ and effective temporal summation, and are powerful enough to delay or completely block spiking in postsynaptic SPNs (Koós and Tepper, 1999). This is in contrast to the axon collateral synapses between SPNs (Tunstall et al., 2002), which typically evoke significantly smaller IPSPs/IPSCs than FSI-evoked synaptic responses when recorded somatically (Koós et al., 2004; Tepper et al., 2004, 2008; Gustafson et al., 2006) due to a combination of predominantly distal synaptic locations (88\%; Wilson and Groves, 1980) and relatively few synaptic (2-3) connections made by each SPN on each postsynaptic SPN (Koós et al., 2004). Note, however, that a recent report described sixfold larger collateral synaptic currents in a small fraction of the SPNSPN pairs recorded (Tecuapetla et al., 2009). Powerful inhibition of SPNs by FSIs in vivo has also been demonstrated during periods of increased cortical activity (Mallet et al., 2005).

The probability of a synaptic connection between a FSI and a SPN within the radius of the FSIs axonal field is extremely high, ranging from $25 \%$ in the first studies in rat slices (Koós and Tepper, 1999) to between 48 and $75 \%$ in more recent experiments in mouse slices (Gittis et al., 2010; Planert et al., 2010). These numbers are significantly greater than the connection probability between pairs of SPNs, which is consistently reported to be between 10 and 20\% (Czubayko and Plenz, 2002; Tunstall et al., 2002; Koós et al., 2004; Taverna et al., 2004).

Striatal FSIs make synapses onto both direct and indirect pathway SPN. The biophysical properties of the synaptic contacts do not differ and exhibit short-term depression. Further, single FSI often make synapses with both types of SPN (Gittis et al., 2010; Planert et al., 2010; see Figure 2). In addition to synapses with SPNs, FSIs have been shown to make functional synaptic connections with other FSIs, but not with cholinergic or PLTS interneurons. Unlike SPN axon collateral interactions, which are almost exclusively unidirectional (see Tepper et al., 2008 for review), the probability of reciprocal synaptic connections between pairs of FSIs is high (Gittis et al., 2010).

It is now generally accepted that striatal $\mathrm{PV}^{+}$FSIs are the major components of a powerful, feedforward inhibition that regulate spike timing in SPNs, thereby regulating striatal output.

\section{PHARMACOLOGY}

Bath application of DA or selective $\mathrm{D}_{1} / \mathrm{D}_{5}$ dopamine agonists induces depolarization and an increase in input resistance in striatal FSI in brain slices, both blocked by $\mathrm{SCH} 23390$, a selective $\mathrm{D}_{1} / \mathrm{D}_{5}$ dopamine antagonist (Bracci et al., 2002). A subsequent study with $\mathrm{D}_{1}$ knockout mice revealed that this effect was due to activation of postsynaptic $\mathrm{D}_{5}$ receptors located on the FSI (Centonze et al., 2003). The 
A MSN D1-EGFP (-)

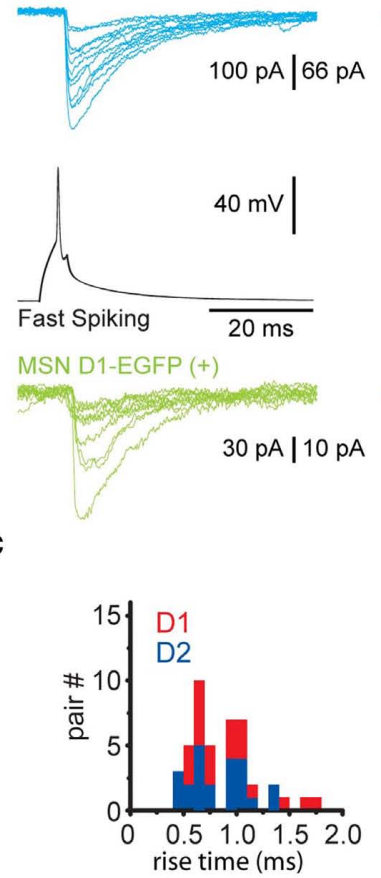

西

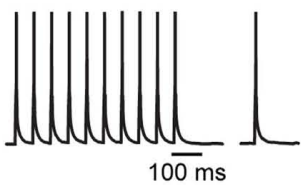

$10 \overline{\mathrm{ms}}$
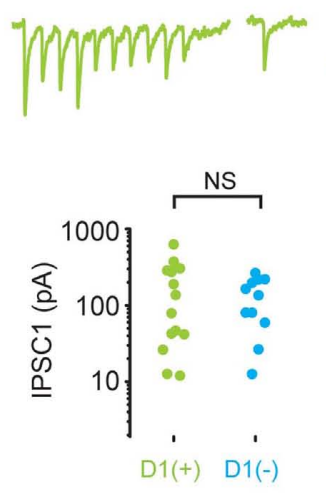

FIGURE 2 | Single FS interneurons evoke large IPSCs in both direct and indirect pathway SPNs. (A) Representative recording from two SPNs that are postsynaptic to the same FSI (black traces) in a striatal slice from a BAC transgenic $D_{1}$-EGFP mouse. The green traces are from the EGFP-D,-expressing striatonigral SPN shown in (B). The blue traces are from another SPN that does not express EGFP and is therefore a D2-expressing striatopallidal neuron. Note similar characteristics (IPSC amplitude, extremely reliable [zero failures] synaptic transmission, short term depression) of each synaptic connection. (B) Upper

B

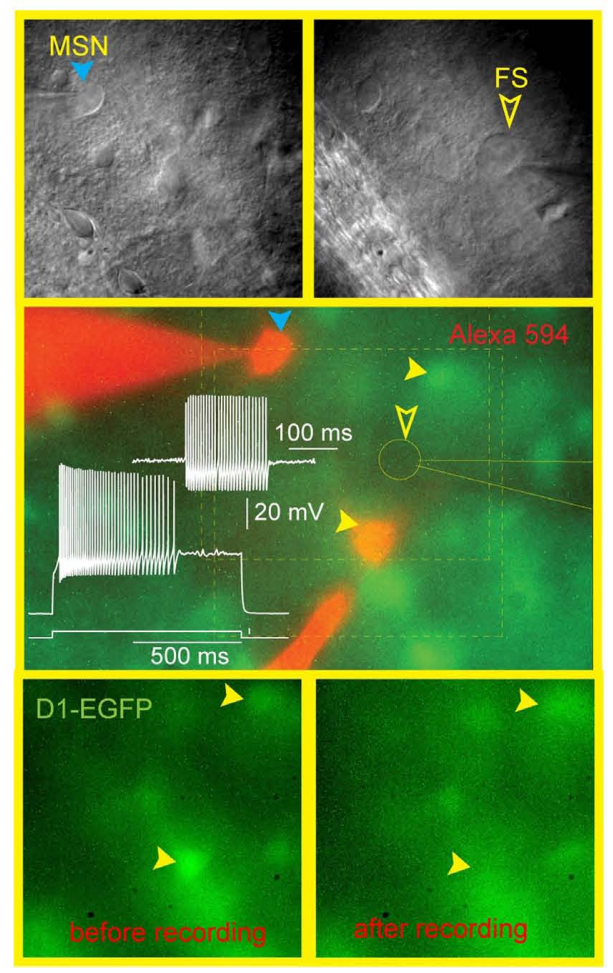

panels show the striatopallidal SPN and FSI and patch pipettes visualized with DIC. Middle panel shows electrophysiological identification of the FSI. The striatopallidal neuron is recorded and stained with a patch pipette containing Alexa 594 (orange). Bottom panels show the disappearance of the EGFP from the striatonigral neuron as a result of dialysis during recording. (C) Summary data from all triple recordings consisting of one FSI presynaptic to a striatonigral ( $n=14)$ and striatopallidal $(n=11)$ neuron reveals no difference in IPSC rise time or amplitude in striatonigral and striatopallidal neurons. ionic mechanism of the depolarization remains unknown. In vivo, systemic application of amphetamine leads to a dose-dependent increase in firing rate of most FSIs whereas the selective DA $\mathrm{D}_{2}$ receptor antagonist, eticlopride, produced a consistent increase in FSI firing rate (Wiltschko et al., 2010). As there are apparently no postsynaptic D2-class receptors on striatal FSIs, these effects are likely due to presynaptic modulation of GABAergic inputs, possibly originating from the GP (Wiltschko et al., 2010), although this remains to be demonstrated directly.

ACh has a dual action on striatal FSIs. In vitro, these interneurons are strongly depolarized and induced to fire by bath or local application of carbachol, a response that is sustained for the duration of application. The response is completely blocked by mecamylamine but not by methyllycaconitine, indicating the involvement of a non-desensitizing, rapidly acting nicotinic receptor distinct from Type- 1 receptors.

In addition to the nicotinic excitation, the FSI-SPN synapse is subject to powerful presynaptic inhibition by a pirenzapinesensitive muscarinic receptor. It has been suggested (Koós and Tepper, 2002) that this presynaptic effect predominates during periods of cortical arousal when ACh levels are high (Abercrombie and DeBoer, 1997) and FSIs are firing rapidly (Mallet et al., 2005).

\section{SOM/NOS/NPY+ INTERNEURONS}

Originally, the GABAergic nature of these neurons was a matter of some debate, with early studies making a clear distinction between $\mathrm{SOM}^{+}$interneurons and GABAergic interneurons (e.g., Lenz et al., 1994) because these neurons did not appear to express either GAD mRNA (Chesselet and Robbins, 1989) or GAD ${ }_{67}$ or GABA immunoreactivity (Kubota et al., 1993). A subsequent experiment in which rats were pretreated with colchicine to block axonal transport and increase somatic levels of proteins synthesized in the soma revealed that striatal $\mathrm{NOS}^{+}$neurons were also immunoreactive for $\mathrm{GAD}_{67}$. A later EM study showed that the synaptic terminals of these neurons were also immunoreactive for GABA (Kubota and Kawaguchi, 2000), ending the controversy once and for all.

Several early immunocytochemical experiments indicated that striatal $\mathrm{SOM}^{+}$interneurons were also immunoreactive for NPY (formerly referred to as avian pancreatic polypeptide; Vincent and Johansson, 1983; Vincent et al., 1983) and the enzyme NADPHdiaphorase, which is equivalent to NOS (Hope et al., 1991), but not for PV or ChAT, thus forming a distinct subtype of striatal GABAergic interneuron (Fujiyama and Masuko, 1996; Gerfen and Wilson, 1996). For the remainder of this review, this neurochemically defined striatal interneuron subtype will be referred to as NPY interneurons. 


\section{NEUROCYTOLOGY}

SOM/NPY/NOS ${ }^{+}$interneurons are medium sized, with round, polygonal, or fusiform somata with diameters ranging from 9 to $25 \mu \mathrm{m}$, making them on average the second-largest striatal neuron after the large aspiny cholinergic interneuron. Typical NPY neurons emit from 3 to 5 thick, aspiny mostly non-varicose proximal dendrites that branch within 30-50 $\mu \mathrm{m}$ of the cell body and taper rapidly, becoming more varicose in the distal regions. The entire arborization is relatively simple, branching sparsely and extends to a diameter of about $600 \mu \mathrm{m}$ (DiFiglia and Aronin, 1982; Vincent and Johansson, 1983; Aoki and Pickel, 1988; Kawaguchi, 1993).

At the electron microscopic level, SOM/NPY/NOS ${ }^{+}$interneurons are characterized by a deeply indented nuclear membrane and a rich cytoplasm, like the $\mathrm{PV}^{+} \mathrm{GABAergic}$ interneurons but in marked contrast to SPN (DiFiglia and Aronin, 1982; Aoki and Pickel, 1988) which exhibit smooth, non-indented nuclear envelopes (Wilson and Groves, 1980).

The axonal arborization of SOM/NPY/NOS ${ }^{+}$is unique among striatal interneurons. It is the least dense axonal arborization of all striatal neurons (although there are exceptions - see Figure $\mathbf{1}$ in
Kubota and Kawaguchi, 2000) and also the largest in overall extent, and consists largely of long, sparsely branching axons extending in straight lines for up to $1 \mathrm{~mm}$ (Kawaguchi, 1993; Kubota and Kawaguchi, 2000). At least some of the axons are myelinated (DiFiglia and Aronin, 1982).

Kawaguchi (1993) reported that a PLTS neuron intracellularly labeled with biocytin appeared to possess two axons, as previously reported for a medium-sized aspiny striatal interneuron in a Golgi preparation (Takagi et al., 1984a). We have encountered the same phenomenon in a mouse PLTS interneuron as shown in Figure 3.

\section{INTRINSIC ELECTROPHYSIOLOGICAL PROPERTIES}

Kawaguchi (1993) obtained whole cell recordings from neurons in slices from juvenile rats that exhibited electrophysiological characteristics that were readily distinguishable from those of SPNs and FSIs. The most characteristic attributes of these neurons were the presence of a low threshold $\mathrm{Ca}^{2+}$ spike (LTS), a very high input resistance ( $>600 \mathrm{M} \Omega$ ), a depolarized resting membrane potential
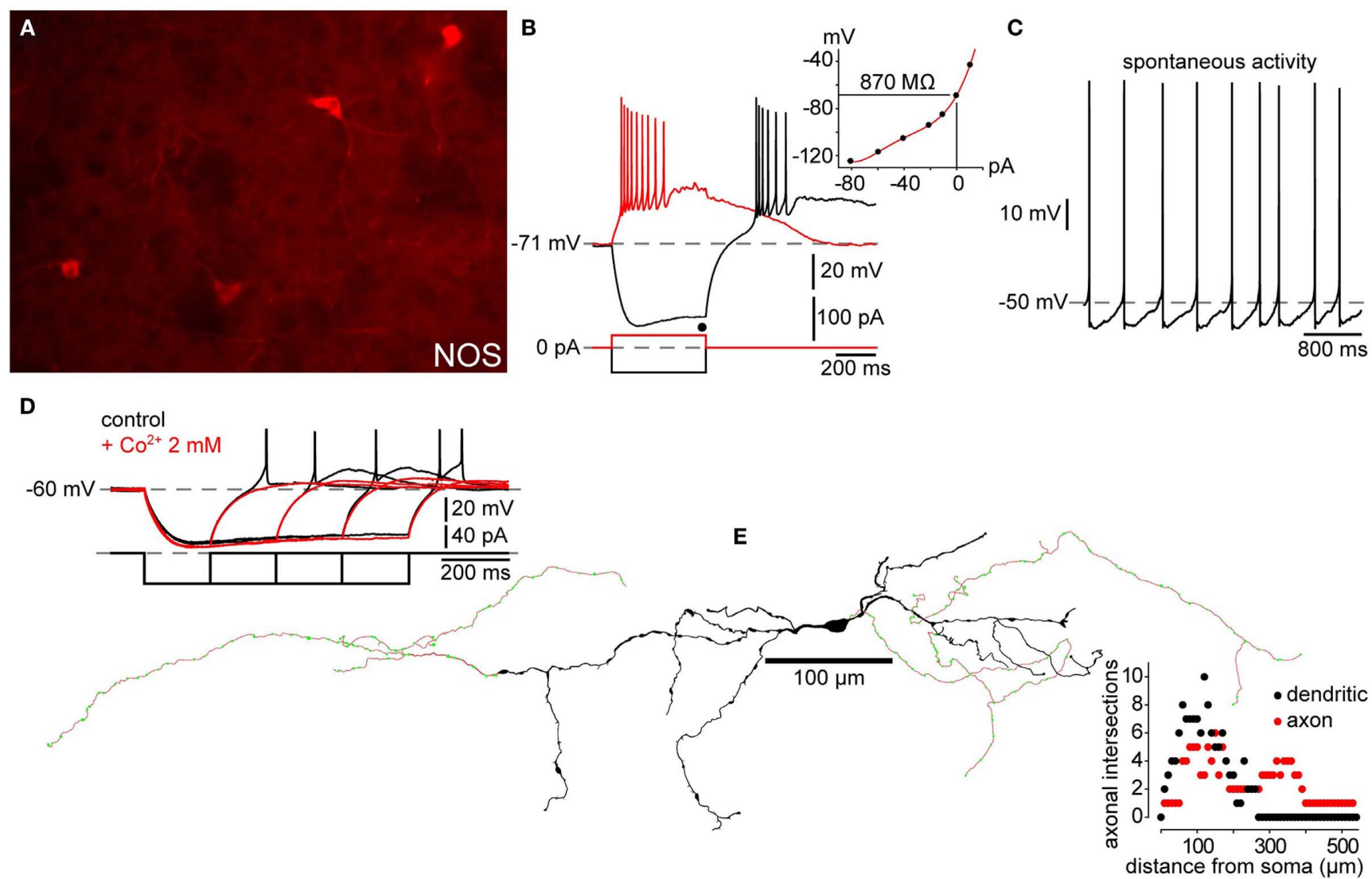

FIGURE 3 | Electrophysiology and morphology of striatal SOM/NPY/NOS interneurons. (A) Immunofluorescence photomicrograph of $\mathrm{NOS}^{+}$mouse striatal interneurons. (B) Passive electrophysiological properties of a NPY+ interneuron from a striatal slice from a BAC transgenic EGFP-NPY+ mouse shows the characteristic relatively depolarized resting membrane potential, LTS following cessation of a hyperpolarizing current injection (black trace), I and, prolonged depolarizing plateau potentials elicited from rest by depolarizing current injection (red trace) and following the rebound LTS after a hyperpolarizing current injection. Inset: IV curve from data in
(B) shows typical high input resistance of striatal PLTS interneurons, even at maximum of $I_{\mathrm{h}}$ activation (black dot). (C) Spontaneous activity typical of many SOM/ NPY/NOS interneurons. (D) Rebound depolarizations display the time dependent de-inactivation, all-or none nature and blockade by $\mathrm{Co}^{2+}$ characteristic of a LTS. (E) Reconstruction of a biocytin filled PLTS interneuron shows typical varicose dendritic arborization with a few sparsely scattered spines. Note the few arborizations in both, axon and dendrites (Sholl plot). This particular example was selected because it had two distinct axons emerging from opposite poles of the soma. 
(approximately $-56 \mathrm{mV}$ ) and the expression of long-lasting plateau potentials following depolarization from rest, in rebound from strong hyperpolarizing current injections or in response to strong excitatory synaptic inputs. These neurons exhibited long duration action potentials ( $1 \mathrm{~ms}$ at half amplitude). Due to the LTS and the persistent depolarizing plateau potentials, these neurons were termed PLTS interneurons (Kawaguchi, 1993) and were subsequently shown to be the $\mathrm{SOM}^{+}$neurons described in the immunocytochemical studies reported above. Subsequent observations in adult rats and mice replicated these findings (Kawaguchi et al., 1995; Kubota and Kawaguchi, 2000; Centonze et al., 2002, 2003; Ibáñez-Sandoval et al., 2010). Although not mentioned in the original reports, a significant proportion ( 30 out of 44 cells) of the PLTS neurons in our mouse slices exhibited tonic spontaneous activity (Ibáñez-Sandoval et al., unpublished). The typical physiological characteristics of striatal PLTS interneurons are illustrated in Figure 3.

Although it was originally assumed that the SOM/NOS/NPY interneurons represented a single population of neurons, more recent stereological cell counting has revealed slightly different numbers for $\mathrm{SOM}^{+}$neurons $(21,300 /$ striatum $=0.8 \%$ of the total $)$ and $\mathrm{NPY}^{+}$neurons (0.57\%; Rymar et al., 2004). Furthermore, a multiple immunocytochemical labeling study concluded that nearly $25 \%$ of the striatal interneurons that expressed various combinations of SOM, NOS, or NADPH diaphorase were not immunoreactive for NPY (Figueredo-Cardenas et al., 1996). Therefore, there appear to be at least three distinct subpopulations of striatal SOM/NPY/NOS ${ }^{+}$interneurons, each expressing different combinations of these three markers. Whether these all subpopulations express the same "classical" electrophysiological phenotype of PLTS interneurons described above is unknown at present.

\section{AFFERENT CONNECTIVITY}

Previous anatomical work showed that PLTS interneurons receive numerous synaptic contacts on their proximal dendrites from both cholinergic and dopaminergic axons, as well as onto their distal dendrites, which receive asymmetric synaptic inputs from the cortex (Kubota et al., 1988; Vuillet et al., 1989a,b, 1992). GABAergic synaptic inputs originating from the globus pallidus were also demonstrated ultrastructurally using juxtacellular labeling and NOS immunocytochemistry (Bevan et al., 1998). The synaptic inputs to NPY neurons were also examined electrophysiologically by Partridge et al. (2009) using a BAC-NPY-GFP transgenic mouse strain and by Gittis et al. (2010) using BAC-Lhx6-GFP transgenic mice. These experiments demonstrated AMPA and NMDA receptor mediated cortical glutamatergic inputs that were relatively weak compared to the inputs of SPNs and $\mathrm{GABA}_{\mathrm{A}}$ receptor mediated inhibitory inputs comparable to those of SPNs (Partridge et al., 2009; Gittis et al., 2010).

\section{EFFERENT CONNECTIVITY}

Not surprisingly, the major efferent target of PLTS interneurons is the SPN. Axon terminals form symmetric synapses, mostly on the distal regions of the dendrites and on spines, largely avoiding the soma (DiFiglia and Aronin, 1982; Aoki and Pickel, 1988; Vuillet et al., 1989a,b; Kubota and Kawaguchi, 2000). NPY+ boutons have also been observed to make symmetric contact with cholinergic interneurons, but synapses between $\mathrm{NPY}^{+}$neurons have not been reported (Vuillet et al., 1989a,b, 1992).

Despite the anatomical evidence cited above, in a recent in vitro paired recording study, in contrast to $\mathrm{PV}^{+}$interneurons, PLTS interneurons were found to evoke only sparse (2/60) and relatively weak GABAergic IPSCs in SPNs (Gittis et al., 2010). In this study, PLTS interneurons were first visually identified in slices from BAC transgenic mice engineered to express EGFP in neurons expressing the homeobox protein Lhx6, a marker for interneurons arising from the ganglionic eminence including $\mathrm{PV}^{+}, \mathrm{CR}^{+}$, and SOM/NPY/NOS interneurons (Marin et al., 2000). No IPSCs were observed in postsynaptic PLTS, FSI, or cholinergic interneurons. Whereas a sparser efferent connectivity than the $\mathrm{PV}^{+}$interneurons is consistent with the much less dense and elaborate axonal arborization, the almost complete absence of postsynaptic responses is not.

One possible explanation for the lack of synaptic responses is that the principal neuroactive substance released from PLTS interneurons may not be GABA. As reviewed above, in contrast to the $\mathrm{PV}^{+}$interneurons, PLTS interneurons express far lower levels of GABA and GAD. Perhaps the principal function of these neurons is to release SOM, NOS, and/or NPY, all of which could exert slower neuromodulatory effects on their postsynaptic targets rather than fast synaptic effects. For example, SOM has been shown to exert a potent presynaptic inhibition on GABA release at SPN-SPN synapses (Lopez-Huerta et al., 2008).

\section{PHARMACOLOGY}

Like the $\mathrm{PV}^{+}$FSIs, the PLTS interneurons are excited by D1-class agonists through D1 family dopamine receptors eliciting depolarization and action potential firing in vitro (Centonze et al., 2002). Interestingly, as is the case with FSIs, the excitatory effect of dopamine on PLTS neurons was also absent in D1 receptor knock out mice indicating the involvement of D5 receptors. In addition, indirect cholinergic effects through $\mathrm{M}_{2}$ muscarinic acetylcholine receptors have also been reported (Bernard et al., 1998).

\section{$\mathrm{CR}^{+}$INTERNEURONS}

Of the three classically recognized striatal GABAergic interneurons, by far the least is known about the CR interneuron. Although they make up $0.5 \%$ of striatal neurons based on stereological cell counts of immunostained material in rat, just slightly less than the number of $\mathrm{PV}^{+}$neurons (Rymar et al., 2004), our knowledge of these interneurons is limited to what can be seen in immunostained material, since they have never been recorded and intracellularly labeled. In primates including humans, the proportion of $\mathrm{CR}^{+}$neurons is much greater than in rodents and $\mathrm{CR}^{+}$interneurons outnumber $\mathrm{PV}^{+}$and SOM/NPY interneurons by 3 or 4 to 1 (Wu and Parent, 2000). No EGFP-CR ${ }^{+}$transgenic mice are currently available, and so there have been no successful attempts thus far to correlate a set of physiological properties with the $\mathrm{CR}^{+}$ phenotype as has been done for other striatal interneurons. The lack of intracellular labeling has also resulted in only a very limited description of the axonal arborization. The lack of data on CR interneurons underscores the power and utility of transgenic mice that selectively express EGFP under the control of a single, specific promotor. 
Early studies in rats described CR-expressing interneurons as medium sized aspiny neurons, 12-20 $\mu \mathrm{m}$ in diameter that issued a small number of smooth, aspiny dendrites that branch sparingly and taper into thin, varicose processes (Bennett and Bolam, 1993; see Figure 4). However, subsequent studies in rats and primates consistently describe at least three or four morphologically distinct types of striatal $\mathrm{CR}^{+}$neurons, ranging from small to large in somatic size (Prensa et al., 1998; Schlosser et al., 1999; Wu and Parent, 2000; Rymar et al., 2004). Indeed, our immunocytochemical studies have revealed the existence of at least three morphologically distinct types of striatal CR interneurons in mouse striatum as shown in Figure 4.

Neonatal hypoxia results in the neurogenesis of $\mathrm{CR}^{+}$striatal interneurons in rats that persists for at least 5 months after induction. Interestingly the neurogenesis appears limited to the $\mathrm{CR}^{+}$ interneurons since there is no neurogenesis of striatal neurons that express markers for any of the other striatal interneurons or projection neurons (Yan et al., 2008).

\section{TH+ INTERNEURONS}

Dubach et al. (1987) first described striatal neurons immunoreactive for $\mathrm{TH}\left(\mathrm{TH}^{+}\right)$in the caudate nucleus of three normal monkeys. The vast majority of these neurons were actually outside the borders of the caudate and putamen, and were located in the white matter ventral to the striatal neuropil. Of the neurons actually located within the striatum, most were restricted to a narrow band in the dorso-medial periphery of the caudate nucleus. These monkey $\mathrm{TH}^{+}$neurons were reported to be bipolar and $8-12 \mu \mathrm{m}$ in diameter. Although no rigorous attempt to count the number of striatal $\mathrm{TH}^{+}$neurons was made,
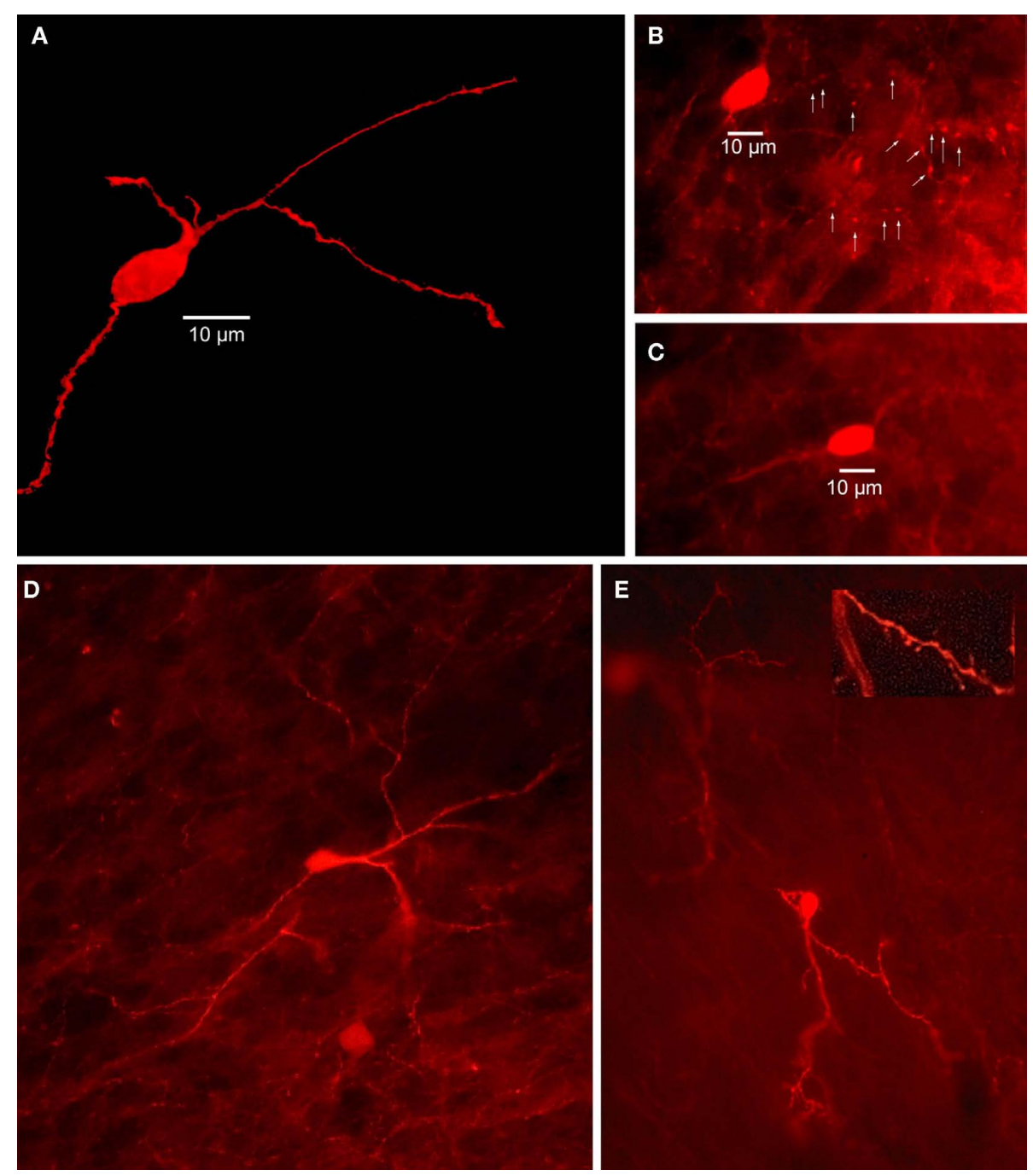

FIGURE 4 | Striatal calretinin immunopositive interneurons.

(A) Two-dimensional projection of 40 deconvolved $1 \mu \mathrm{m}$ optical sections through mouse striatum immunostained for a Type / CR interneuron shows a single medium sized, aspiny $\mathrm{CR}^{+}$interneuron. (B) Immunofluorescence photomicrograph of a single section containing an aspiny medium-sized mouse immunostained striatal $\mathrm{CR}^{+}$interneuron. Arrowheads point to axonal varicosities. (C) Another immunostained aspiny Type / $\mathrm{CR}^{+}$interneuron. (D) Immunostained brightly fluorescent aspiny Type // $\mathrm{CR}^{+}$interneuron just above a less brightly fluorescent Type / $\mathrm{CR}^{+}$interneuron. Note smaller soma of Type // neuron and more high branched dendritic arborization. (E) Least common type of $\mathrm{CR}^{+}$interneuron is the Type III, intensely fluorescent and spiny. 
the authors estimated that they numbered in the "tens of thousands." Identical immunostaining protocols applied to mouse and rat striatum in the same study failed to find any striatal or peristriatal $\mathrm{TH}^{+}$neurons. Interestingly, when the material from these monkeys was subjected to fluorescence histochemistry, only 5-10 fluorescent neurons were observed in the caudate and putamen (Dubach et al., 1987).

In contrast, a subsequent immunostaining experiment in rat striatum did reveal the existence of a very small number $\mathrm{TH}^{+}$neurons (7-19 per striatal hemisphere) in control animals. These neurons were 10-20 $\mu \mathrm{m}$ in diameter, multipolar, and exhibited sparse spines on some dendrites. Interestingly, the number of $\mathrm{TH}^{+}$neurons increased by a factor of $2-4$ times following dopaminergic denervation (Tashiro et al., 1989b), suggesting that the expression of TH in these neurons may be under the control of ambient DA levels.

Subsequently, many studies from different laboratories have confirmed the existence of neurons that could be immunostained with different monoclonal or polyclonal antibodies directed against $\mathrm{TH}$ in mouse, rat, monkey, and man. However, there remained considerable controversy regarding the number of striatal $\mathrm{TH}^{+}$neurons, their identification as interneurons or projection neurons, their morphology, species dependence and other factors (Betarbet et al., 1997; Meredith et al., 1999; Mao et al., 2001; Palfi et al., 2002; Jollivet et al., 2004; Cossette et al., 2005; Mazloom and Smith, 2006; Porrit et al., 2006; Tande et al., 2006; Huot et al., 2007; Darmopil et al., 2008).

Recently we have been able to resolve many of these controversies by using genetically modified mice that express EGFP under the control of the endogenous $\mathrm{TH}$ regulatory sequences $(\mathrm{Tg}$ (Th-EGFP) 1Gsat/Mmnc; Gong et al., 2003). These have allowed us to visualize striatal $\mathrm{TH}^{+}$neurons in brain slices and target them for whole cell recording and biocytin labeling which allowed us to study the electrophysiology and anatomical properties of striatal EGFP-TH ${ }^{+}$neurons (Ibáñez-Sandoval et al., 2010). In that article we reported the existence of four electrophysiological distinct types of striatal EGFP-TH ${ }^{+}$neurons, which were named: Type I, Type II, Type III, and Type IV. After electrophysiological characterization, biocytin-stained EGFP- $\mathrm{TH}^{+}$neurons were reconstructed and described neuroanatomically.

\section{NEUROCYTOLOGY}

Striatal EGFP-TH ${ }^{+}$of all four subtypes neurons exhibited medium sized somata ( width $=15.2 \pm 0.6 \mu \mathrm{m}$ and height $=10.8 \pm 0.4 \mu \mathrm{m}$ ), which were most frequently round or ovoid for Types $I I-I V$, but often polygonal for Type I. These neurons emitted at least two to four aspiny and varicose primary dendrites (88\%). Estimates from unbiased stereology showed the number of EGFP-TH ${ }^{+}$interneurons per striatum was $2684+1216(n=6)$, a number much greater than that in most previous, non-quantitative estimates of striatal $\mathrm{TH}^{+}$neurons labeled by immunocytochemistry in rodents (Tashiro et al., 1989b; Mao et al., 2001; Busceti et al., 2008).

Occasionally (in $12 \%$ of stained neurons) the dendrites from Type I cells exhibited moderately dense, thick stick-like appendages that appeared to lack distinct spine heads (see Figure 5). Nevertheless, these neurons could be readily distinguished from SPNs on morphology alone, and as described below, their very distinct electrophysiological properties. These data are consistent with some of the previous reports based on $\mathrm{TH}^{+}$immunocytochemistry

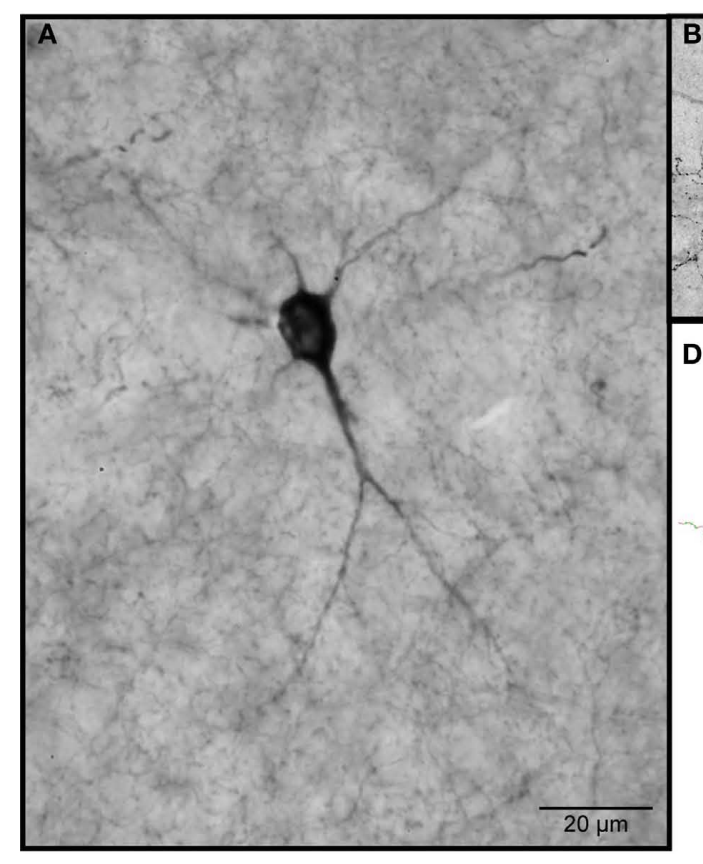

FIGURE 5 | Striatal tyrosine hydroxylase immunopositive interneurons. (A) TH-Immunoreactive aspiny striatal neuron from a normal rhesus macaque monkey. (B) A Type / striatal EGFP-TH+ neuron stained with biocytin during whole cell recording from a striatal slice from an EGFP-TH+ mouse. The dendrites branch infrequently and exhibit sparse, spine-like

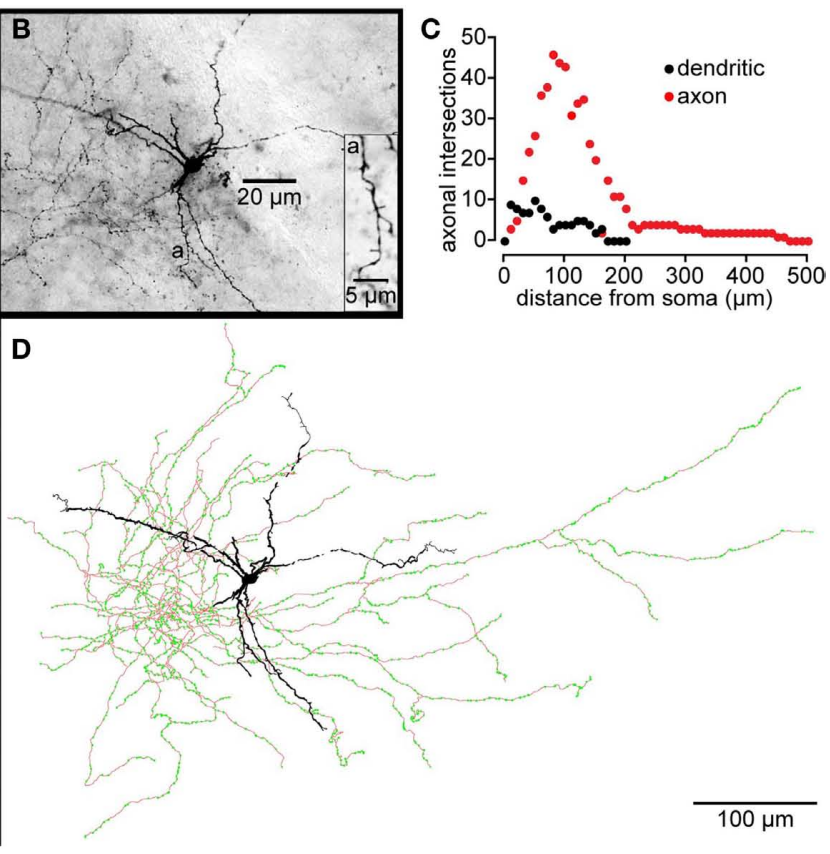

appendages (a). (C) Shows a Sholl plot of the reconstructed neuron revealing the extents and close overlap of the dendritic and axonal arborizations. (D) Drawing tube reconstruction of the neuron shown in (B). Note the dense axonal arborization studded with varicosities (green dots), presumably axonal boutons. 
(Dubach et al., 1987; Betarbet et al., 1997; Cossette et al., 2004, 2005; Mazloom and Smith, 2006; Huot and Parent, 2007) but are in sharp contrast to others that claimed the striatal $\mathrm{TH}^{+}$neurons to be a subpopulation of SPNs (e.g., Tashiro et al., 1989a,b; Darmopil et al., 2008). Types II, III, and IV could not be distinguished on morphological grounds.

The biocytin labeling of EGFP-TH ${ }^{+}$neurons allowed the first descriptions of their axonal arborizations (Ibáñez-Sandoval et al., 2010). These data revealed that for all four cell types, the axon emerged from the soma or proximal dendrite and branched almost immediately forming a dense local axon collateral plexus that occupied a volume coextensive with and sometimes extending beyond the dendritic tree of the issuing neuron (Figure 5). The collaterals were highly branched and studded with large numbers of prominent varicosities. None of the filled cells exhibited a single axonal branch that was larger than the rest or that could be clearly identified as the main axon. These data are consistent with our retrograde labeling data that failed to show any EGFP- $\mathrm{TH}^{+}$neurons retrogradely labeled from large Fluorogold injections in substantia nigra and GP.

Immunocytochemical studies using rat, monkey, and human material have demonstrated the presence of the GABAergic markers, $\mathrm{GAD}_{65}$ and/or $\mathrm{GAD}_{67}$ (Betarbet et al., 1997; Cossette et al., 2005; Mazloom and Smith, 2006; Tande et al., 2006; San Sebastián et al., 2007), dopaminergic markers including the dopamine transporter (DAT, Betarbet et al., 1997; Palfi et al., 2002; Cossette et al., 2004; Tande et al., 2006), and the synthetic enzyme a-aromatic amino acid decarboxylase (AACD, Mura et al., 1995, 2000; Meredith et al., 1999; Lopez-Real et al., 2003), and less frequently CR (Mura et al., 2000; Cossette et al., 2004, 2005; Tande et al., 2006) in $\mathrm{TH}^{+}$interneurons. Moreover, a small number of single cell RT-PCR experiments demonstrated in Type $I$ and $I V \mathrm{TH}^{+}$interneurons (the only two types examined with sc-RT-PCR) the expression of an isoform of the obligatory marker of monoamine release, the vesicular monoamine transporter (VMAT), VMAT-1 and the apparent absence of the more common isoform, VMAT-2, expressed by mesencephalic DA neurons (Ibáñez-Sandoval et al., 2010). Importantly, $\mathrm{TH}^{+}$striatal interneurons were directly demonstrated to be distinct from PV, NOS, or CR expressing neurons in BAC-TH-EGFP mice (IbáñezSandoval et al., 2010). In addition, these interneurons are present in both the matrix and patch compartments of the striatum, but appear to be more frequent in the matrix in primates (Huot et al., 2007).

The presence of TH immunoreactive neurons in the striatum was a matter of some debate in normal animals (Dubach et al., 1987; Tashiro et al., 1989a,b; Betarbet et al., 1997; Palfi et al., 2002; Cossette et al., 2004, 2005; Tande et al., 2006; Huot et al., 2007). In some studies, striatal $\mathrm{TH}^{+}$neurons were seen only after dopamine denervation (Mura et al., 1995; Meredith et al., 1999; Lopez-Real et al., 2003; Darmopil et al., 2008). A subsequent experiment in which mice were pretreated with colchicine to block axonal transport in an attempt to facilitate somatic accumulation of TH revealed TH immunoreactive neurons in the striatum. It still remains to be clarified if these neurons are able to synthesize and release dopamine.

\section{INTRINSIC ELECTROPHYSIOLOGICAL PROPERTIES}

Electrophysiological recordings of striatal EGFP-TH ${ }^{+}$cells revealed significant heterogeneity among these neurons with respect to their resting membrane potential, membrane potential responses to current injection, input resistance, spontaneous activity, action potential waveform, and maximum firing rate. Based on a highly reproducible pattern of correlations between a number of electrophysiological characteristics four distinct types of $\mathrm{TH}^{+}$interneurons could be distinguished and were named Type I-IV TH interneurons. The most frequently encountered subtype is Type I (60\%) followed by Type IV (21\%), Type II (13\%), and finally the Type III (6\%; Figure 6). The electrophysiological properties that most distinctly identify the Type I interneurons is their extremely high input resistance (346-1500 M $\Omega$ ), their inability to maintain continuous firing during depolarizing current injections and a depolarization induced long-lasting plateau potential that is generated in a self-sustaining manner by a nimodipine sensitive L-type $\mathrm{Ca}^{2+}$ conductance and a flufenamic acid sensitive non-selective cationic conductance $\left(I_{\mathrm{CAN}}\right.$, Figure 6C; Ibáñez-Sandoval et al., 2010). In addition, Type I neurons had action potentials exhibiting highly variable durations (0.4-1.9 ms half amplitude) and fast adaptation. In some cases Type I neurons also exhibited spontaneous fluctuations in membrane potential of $\sim 10 \mathrm{mV}$ in amplitude, that resembled up and down states in spiny neurons (Wilson and Kawaguchi, 1996). Hyperpolarizing current pulses in the majority of Type I cells elicited a membrane potential deflection that could be blocked by ZD7288 $(100 \mu \mathrm{M})$, indicating that it was attributable to activation of $I_{h}$.

In contrast, the Type II cells had somewhat lower input resistances (234-758 $\mathrm{M} \Omega$; Figure 6), and were further distinct in their ability to fire action potentials throughout a depolarizing current injections and by exhibiting higher maximal firing rates (137-265 Hz), and little adaptation (Figure 6C). Type II neurons also had short duration action potentials $(0.3-0.53 \mathrm{~ms})$, and large amplitude after hyperpolarization ( $16-25 \mathrm{mV}$ ). While hyperpolarizing current pulses in almost all of the Type II cells, elicited a HCN channel mediated sag response similar to those of Type I neurons, Type II cells did not exhibit the characteristic plateau potentials of Type I neurons.

The Type III interneurons could be distinguished by the most negative resting membrane potentials $(-89 \mathrm{mV})$ and the lowest input resistance among $\mathrm{TH}^{+}$neurons (150-205 $\mathrm{M} \Omega$ ), which was due to a strong inward rectification present at membrane potentials more negative to $-80 \mathrm{mV}$ (Figure 6). Like Type I cells, the Type III interneurons were incapable of sustained firing in response to large amplitude current pulses but at much lower intensities DC current injection elicited continuous firing (Figure 6C). These neurons also exhibited a nimodipine sensitive plateau potential similar to those of Type I neurons.

Finally, like Type I and Type II cells, Type IV interneurons exhibited high input resistances (235-821 M 2 ) and a HCN channel mediated sag response to hyperpolarizing current injections similar to those in Type I and Type II neurons, but were clearly distinguished from these cell types by exhibiting a low-threshold spike (LTS), that could be elicited at the resting membrane potential by depolarization or when rebounding from hyperpolarizing current injections (Figure 6C). The LTS was accompanied by a short burst of fast action potentials exhibiting intra-burst frequencies in excess of $300 \mathrm{~Hz}$. Type IV neurons had short-duration action potentials (0.4-0.85 ms) as well. 
A

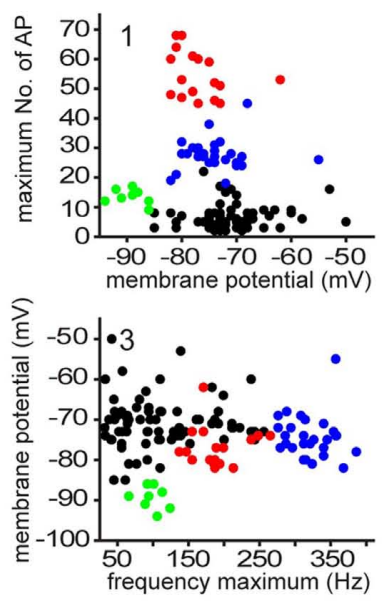

C

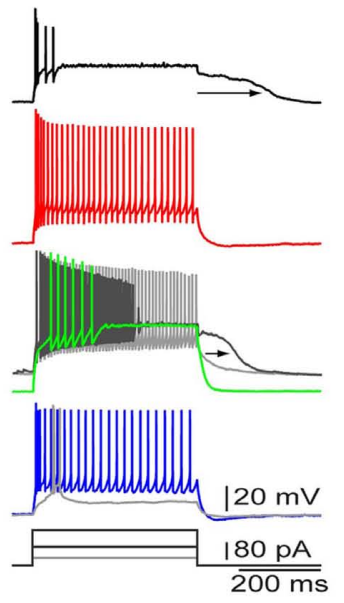

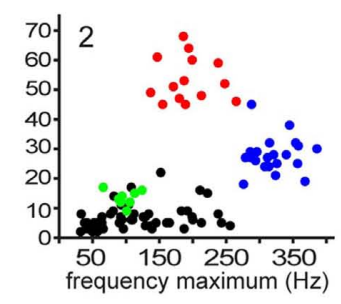

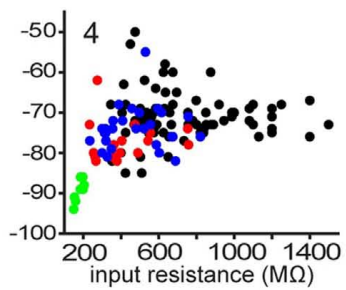

D
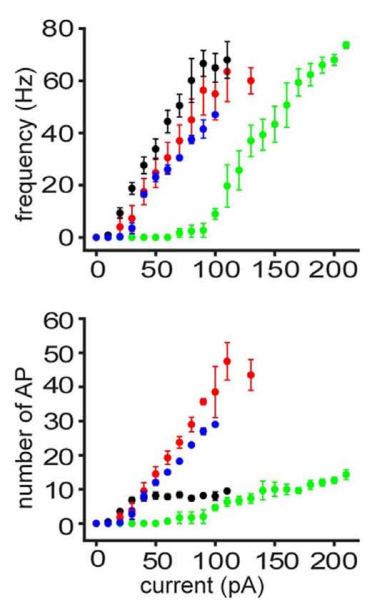

B

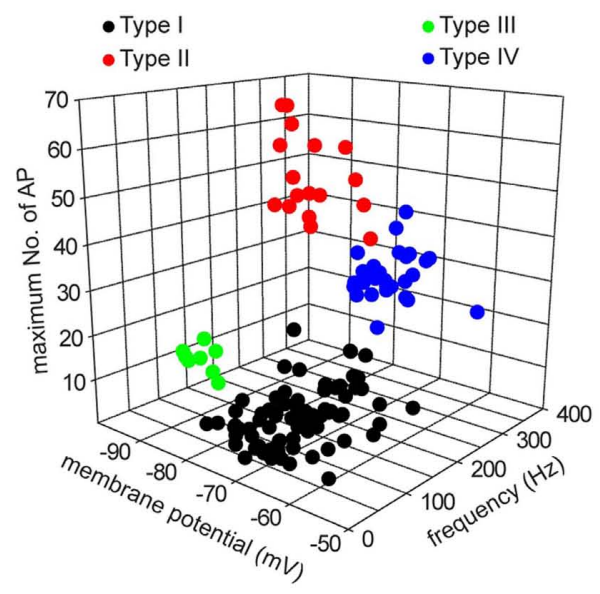

E

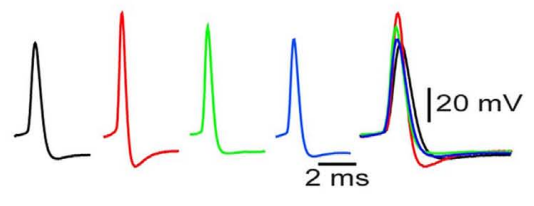

$\mathbf{F}$

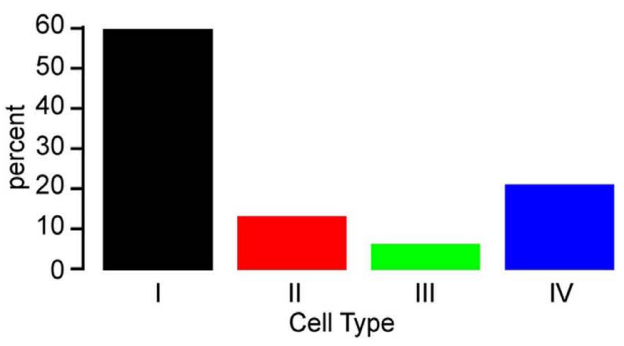

FIGURE 6 | Four different types of striatal EGFP-TH+ neurons in mice. (A) Selected two-dimensional scatter plots of various electrophysiological parameters reveal the separation of striatal EGFP-TH+ neurons into four distinct groups, termed Types I-IV. (B) Clustering of four distinct cell types in one representative three-dimensional scatter plot. (C, D) Voltage response to depolarizing current injection (100 pA), for four striatal $\mathrm{TH}^{+}$neuron types, showing the relationship between injected current and maximum number of spikes evoked or maximum firing rate show clear differences between the four striatal $\mathrm{TH}^{+}$types neurons. Note the plateau potential that was evoked in the Type / (arrow) at rest and in a slightly depolarized Type I/I neuron (arrow), using a stronger depolarizing current injection (180 pA). The Type //I fires throughout the depolarizing current (gray line). In addition, the Type IV interneuron exhibits a clear LTS component in response to a small depolarizing current injection (40 pA), at its resting membrane potential. (E) Averaged action potentials from cell Types I-IV clearly show differences in multiple spike waveform parameters. (F) Histogram showing the distribution of the four EGFP-TH+ cell types.
Unlike Type I, Type II, and Type III neurons, that exhibited electrophysiological properties unlike those of any previously identified cell type in the neostriatum, the Type IV cell closely resembled the LTS interneuron described by Koós and Tepper (1999). However, Type IV $\mathrm{TH}^{+}$neurons as well as the previously described LTS neurons both differed from the NPY/NOS/SOM ${ }^{+}$PLTS neuron described by Kawaguchi and colleagues (Kawaguchi, 1993; Kawaguchi et al., 1995) due to the absence of plateau potentials, lower input resistances and a shorter duration action potentials.

\section{AFFERENT CONNECTIVITY}

Previous anatomical work showed sparse but clearly defined asymmetrical and symmetrical axodendritic synaptic contacts on $\mathrm{TH}^{+}$striatal neurons (Mazloom and Smith, 2006), suggesting that $\mathrm{TH}^{+}$neurons are integrated into the striatal circuit. In this regard, recent studies shown that at least Type I neurons respond to cortical stimulation (IbáñezSandoval et al., 2010), evoking an EPSP that was blocked by $10 \mu \mathrm{M}$ of DNQX, showing the involvement of AMPA/kainate type glutamate receptors. In addition, intrastriatal stimulation evoked a compound response that consisted of $\mathrm{GABA}_{\mathrm{A}}$ receptor mediated inhibitory and AMPA receptor mediated glutamatergic excitatory components. The cellular origin of the excitatory responses has not been determined.

Interestingly, paired recordings showed that both EGFP-TH Type I and Type II interneurons receive GABAergic inhibitory inputs from SPNs (Ibáñez-Sandoval et al., 2010). Single action potentials elicited in SPN evoked a IPSPs in postsynaptic neurons depolarized with current injection that was sufficient in amplitude to delay action potential firing (Figure 7B). The IPSP reversed near to the chloride equilibrium potential (Figure 7A), and could be blocked by bicuculline $(10 \mu \mathrm{M})$, indicating that the IPSPs were mediated by 


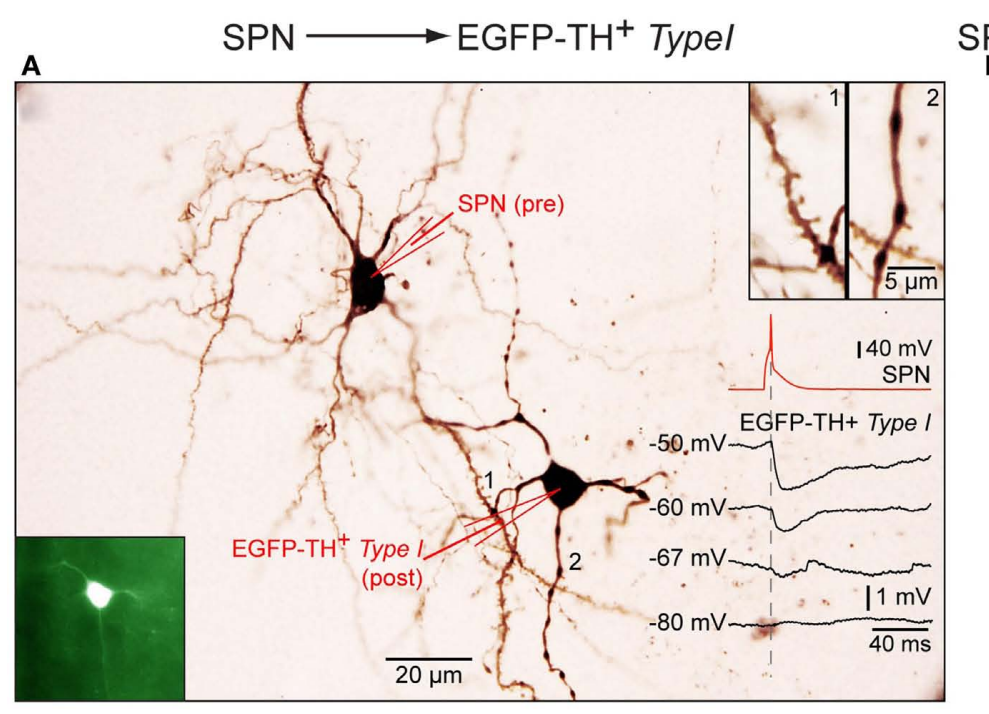

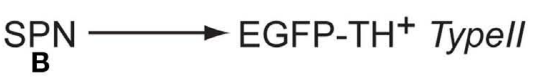

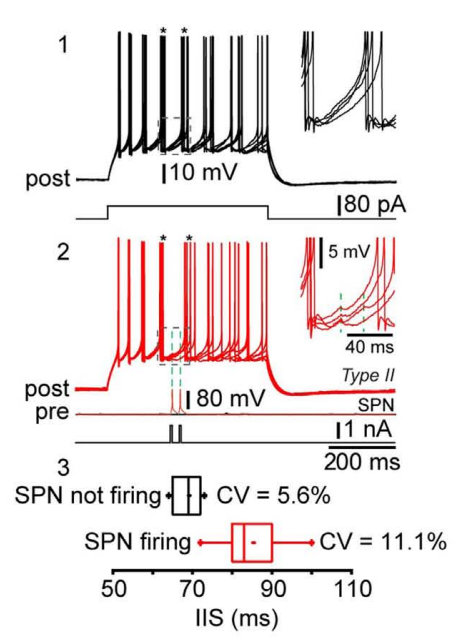

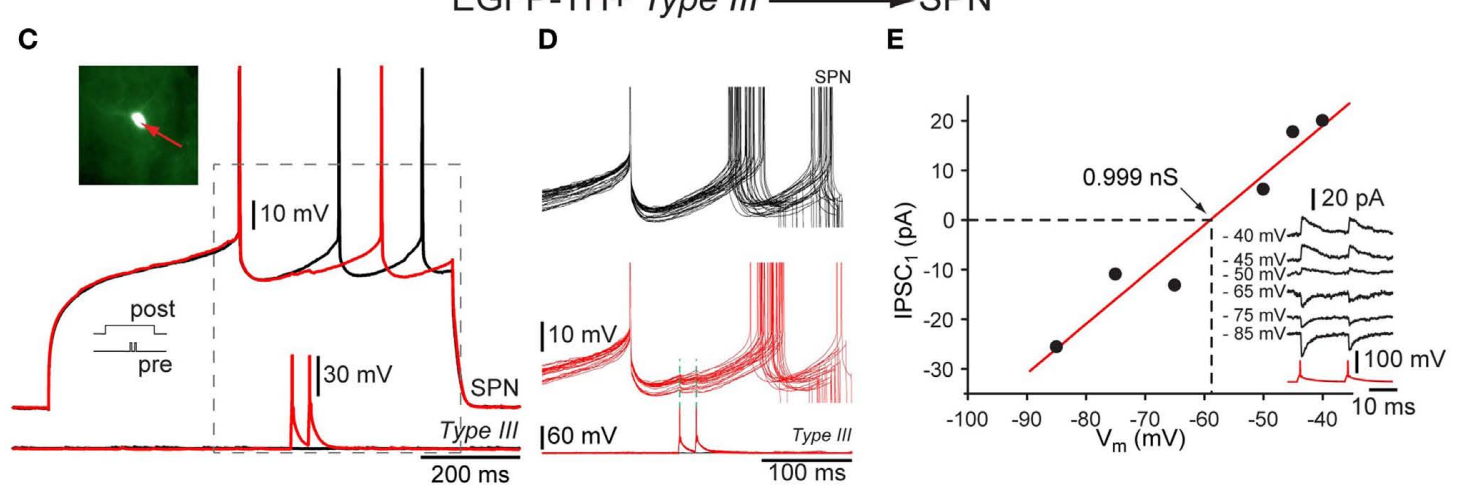

FIGURE 7 | Synaptic interactions of TH interneurons. Whole cell recordings from three connected pairs in which the first pair consisted of a presynaptic SPN and a postsynaptic Type / neuron, the second pair a presynaptic SPN and a postsynaptic Type // interneuron and the third pair of a presynaptic Type /// neuron and a postsynaptic SPN. (A) Photomicrograph showing pair 1 intracellularly labeled with biocytin. Bottom inset: Fluorescence photomicrograph of the postsynaptic Type / EGFP-TH interneuron (bottom left), Top inset: spine laden dendritic segment of the SPN (1) and a varicose dendrite from Type / neuron (2) at higher magnification. Traces show a single action potential in the SPN (red), eliciting IPSPs at different postsynaptic membrane potentials (in black) showing reversal near $-67 \mathrm{mV}$. (B) Depolarization evoked repetitive firing of a Type /I neuron (1, black traces) is interrupted at the time indicated by the asterisks by single spikes of evoked in the presynaptic SPN (2, red traces) Inset shows the IPSP enlarged. Panel 3 shows the cumulative data for the inter-spike intervals with (black) and with presynaptic activity (red). (C) An analogous experiment shows delay in the depolarization induced spiking in an SPN (top red and black traces) by two spikes in the presynaptic Type III interneuron. (D) Higher magnification of the IPSP. Note the reliability of transmission. (E) $I-V$ plot for the synaptic response in (C) and (D) showing a reversal potential as expected for $\mathrm{Cl}^{-}$and providing an estimate of the synaptic conductance $(\sim 1 \mathrm{nS})$.
GABA $_{A}$ receptors (see Ibáñez-Sandoval et al., 2010). These synaptic connections are of particular interest because they represent the only demonstrated fast GABAergic inhibitory input to any striatal interneuron type from SPNs. Furthermore the failure of comparable previous paired recording experiments (Koós and Tepper, 1999) to detect an inhibitory input from SPNs to FSIs suggest the intriguing possibility the $\mathrm{TH}^{+}$interneurons and FSIs play fundamentally different roles in the striatal circuitry.

\section{EFFERENT CONNECTIVITY}

Using paired recordings, Ibáñez-Sandoval et al. (2010) have demonstrated that Type I, II, and III TH interneurons all innervate SPNs and that single presynaptic action potentials often elicit large amplitude
IPSPs in their postsynaptic targets. All three neurons were shown to elicit IPSPs that are mediated by $\mathrm{GABA}_{\mathrm{A}}$ receptors confirming the previous identification of these neurons as GABAergic (Betarbet et al., 1997; Cossette et al., 2005; Mazloom and Smith, 2006; Tande et al., 2006; San Sebastián et al., 2007). The biophysical properties of the connections of the three types of interneurons appear to be heterogeneous. While synapses of Type I and II neurons elicited IPSP/ Cs characterized by relatively large amplitudes and undetectably low failure rates, Type III neurons elicited responses in control condition that were of similar average amplitudes and were not associated with failure rates (Figures 7C-E). All of these inputs were, however, sufficiently strong to delay action potential firing in SPNs depolarized with intracellular current injection (Ibáñez-Sandoval et al., 2010). 

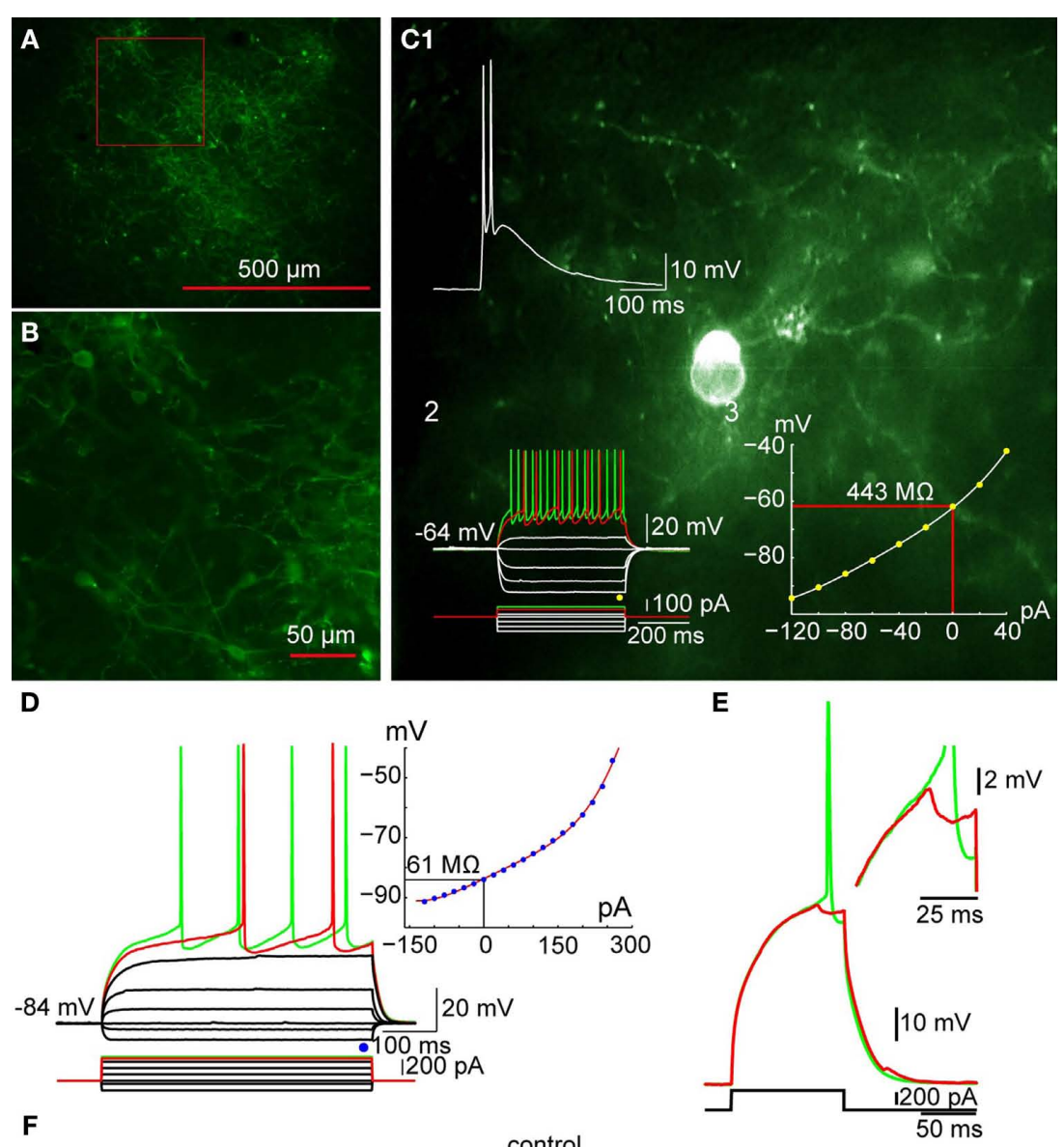

$\mathbf{F}$

control

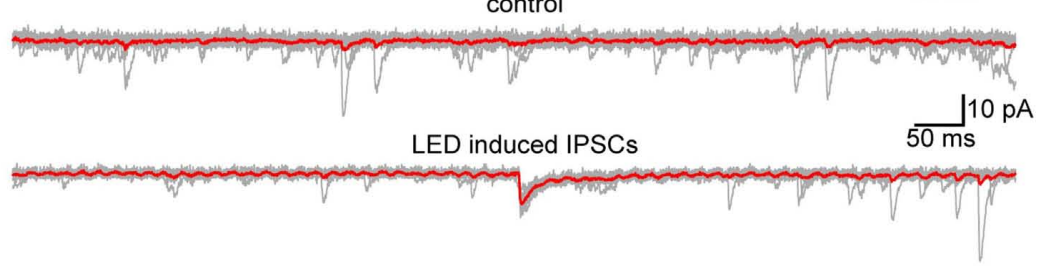

FIGURE 8 | Optogenetic activation of $\mathrm{TH}^{+}$interneurons inhibits SPN firing. (A) Photomicrograph of a parasagittal slice obtained from a TH-Cre mouse injected with AAV-2:EF1:DOI:CHR2-EGFP. Note the large area of infection ( $1 \mathrm{~mm}$, in diameter). (B) Higher magnification of the area indicated by the red rectangle in $\mathrm{A}$ showing numerous $\mathrm{AAV}$-infected $\mathrm{TH}^{+}$interneurons as well as axonal and dendritic processes expressing ChR2-YFP (green).

(C) Photomicrograph of a single ChR2 expressing striatal TH

interneuron recorded in current clamp. C1: A 2-ms pulse of blue light elicits action potentials in the $\mathrm{TH}^{+}$interneuron. $\mathrm{C} 2$ : Responses to injected current identify the trasfected neuron as a Type $/ / \mathrm{TH}^{+}$interneuron. C3: $I-\mathrm{V}$ plot from data in C2 is typical of Type // interneurons. (D) SPN recorded in another ChR2-expressing striatal slice. Inset shows typical $I-V$ characteristics. (E) Action potential firing elicited in the SPN in D with current injection can be blocked by brief ( $2 \mathrm{~ms}$ ) optogenetic stimulation of $\mathrm{TH}^{+}$interneurons and axons. Inset shows the IPSP elicited in the SPN at higher magnification. (F) Current traces obtained without (top traces) and with optical stimulation (bottom traces). $V_{\mathrm{h}}=-80 \mathrm{mV}$. Note the IPSC elicited in the SPN (bottom).
To asses the role of the relative small population of TH interneurons in the inhibitory control of the neostriatum it is important to determine what fraction of SPNs receive input from these source and the strength of inhibition that may be exerted by a concerted activity of this interneuron population. We have conducted preliminary experiments to address this issue using Channelrhodopsin-2 (ChR2) mediated optogenetic activation of genetically targeted TH interneurons.
Fluorescent-tagged ChR2 (ChR2-YFP or ChR2-dTomato) was expressed in $\mathrm{TH}^{+}$interneurons with viral mediated transfer of a Cre/lox controlled transgenes (Tsai et al., 2009) using serotype-2 or 5 adeno-associated virus in BAC transgenic TH-Cre [ $\mathrm{Tg}(\mathrm{Th}$ cre) 12 Gsat mice (Figures 8A,B). Using this method postsynaptic responses to brief optical stimulation of $\mathrm{TH}$ interneurons could be detected in the majority of SPNs (see Figure 8) and these response were able to prevent action potential firing of the postsynaptic 
neuron induced by current injection (Figure 8E). Together the currently available data supports the conclusion that despite their relatively small population $\mathrm{TH}$ interneurons may contribute significantly to the GABAergic inhibitory control of SPNs.

\section{ARE THERE OTHER STRIATAL GABAERGIC INTERNEURONS? CCK+ $^{+}$AND VIP+ NEURONS}

Although a small number of cholecystokinin (CCK) and vasoactive intestinal polypeptide (VIP) aspiny neurons have been described in the striatum of the rat (Takagi et al., 1984b; Theirault and Lamdis, 1987; Hökfelt et al., 1988), as well as CCK neurons in the cat (Adams and Fisher, 1990), nothing is known about their physiological properties or synaptic actions. Both CCK and VIP neurons were reported as medium size (12-17 $\mu \mathrm{m}$; VIP neurons and 10-20 $\mu \mathrm{m}$; CCK neurons), with few primary dendrites (3-5), which branched close to the soma and whose dendrites became varicose and aspiny appearing. Moreover, in contrast to the sparsely distributed VIP neuron population observed in all areas of the striatum (Theirault and Lamdis, 1987), the observation of a very small population of CCK neurons in the cat (Adams and Fisher, 1990) could not be replicated in rat (Gilles et al., 1983) or human material (Schiffmann et al., 1989). In rats, the failure to detect CCK in the striatum persisted even after colchicine treatment (Gilles et al., 1983; Záborszky et al., 1985). Nevertheless, the possibility remains that detection of CCK and VIP interneurons is limited or prevented by low levels of neuropeptide expression or high sensitivity to colchicine. Preliminary single cell RT-PCR analysis of a small number of $\mathrm{TH}$ interneurons suggest that some if these neurons may express CCK and/or VIP implying a possible overlap among these types of interneurons. Genetic reporting and/or targeting methods as well gene expression assays will be essential to clarify the existence and possible roles these neurons in the striatum.

Finally, indirect electrophysiological evidence has been presented recently demonstrating the existence of a GABAergic population of neurons in the neostriatum which are activated by cholinergic interneurons and provide strong feedback inhibition of the same cholinergic interneurons (Sullivan et al., 2008). Since SPNs do not express nicotinic receptors or display nicotinic EPSPs the feedback neurons must be an interneuron. Recordings from eight pairs of FSIs and cholinergic interneurons failed to demonstrate a synaptic innervation in either direction (Tecuapetla et al., unpublished observation, Koós and Tepper, unpublished observations) suggesting that despite of the presence of soma-dendritic nicotinic receptors on FSIs these neurons may not be responsible for the feedback inhibition of SPNs. Further experiments will be required to identify these interneurons.

\section{FUNCTIONAL IMPLICATIONS OF THE DIVERSITY OF STRIATAL INTERNEURONS}

The data reviewed here shows that the neostriatal circuit incorporates an unexpectedly large variety of inhibitory interneurons that exhibit highly specialized intrinsic properties and connectivity. While the existence of such an intricate organization provides in itself a compelling argument for a fundamental role of the local circuitry in the behavioral functions of the striatum, the precise function(s) of interneuronal inhibition in the striatum remain largely unknown. Moreover, it is puzzling why such a large variety of interneurons exists in the striatum and what distinct functions these individually small and in some cases minute neuron populations may serve.
Understanding the information encoded by GABAergic striatal interneurons or other contingencies of their activity is in its infancy. Recently, multiunit recording experiments have provided some information about fast-spiking units (FSUs), which exhibit action potential waveforms and firing properties resembling FSIs. Interestingly, in behaving rats navigating in a baited maze, the activity of these neurons does not systematically co-vary with the animal's position relative to rewarded or otherwise significant locations (Berke, 2008). Instead, in a choice paradigm these neurons fire during choice execution exhibiting specificity to the direction of the movement chosen by the animal. Interestingly, the directional selectivity of FSUs and SPNs were the opposite, suggesting that inhibition from interneurons may contribute to the direction selectivity of the SPN responses. A perhaps related spatial pattern of activation of FSIs was described earlier based on immediate early gene expression used as a reporter of $\mathrm{PV}^{+}$interneuron activity in response to cortical activation (Parthasarathy and Graybiel, 1997).

A highly intriguing possibility is that a major function of at least some interneuron types is the coordination or control of gamma frequency oscillations observed primarily in the ventral parts of the neostriatum and the nucleus accumbens. Gamma oscillations are generated by synchronous periodic activity of GABAergic interneurons which act as a pacemaker for the firing of principal neurons throughout diverse neuronal systems including the neocortex, hippocampus, and thalamus (Freund and Buzsáki, 1996; Traub et al., 1999; Bartos et al., 2007) as well as the insect olfactory system (Laurent, 2002). Although it remains uncertain if gamma oscillations in the ventral striatum are of local origin, spiking activity of FSUs (Berke, 2008; van der Meer and Redish, 2009) and at least under some conditions the activity of presumed SPNs (Popescu et al., 2009; Kalenscher et al., 2010), phase lock to this activity, and the striatal gamma becomes synchronized with a non-zero phase lag to oscillations in the amygdala after conditioning (Popescu et al., 2009), suggesting a significant contribution from local mechanisms. Moreover, in the hippocampus the synchronous oscillatory activity of FSIs is generated by interplay of mutual inhibition and intrinsic resonant properties of interneurons (Traub et al., 1999) and in part through electrotonic coupling (Pais et al., 2003). Remarkably, the same features of dense synaptic interconnections, electrotonic coupling and unique intrinsic properties, including resonance at a gamma frequency range are displayed by FSIs in the striatum (Koós and Tepper, 1999; Bracci et al., 2003; Tepper, 2010), and there is also an overlap of the location of high voltage spindles and FSI units in the dorsal striatum (Berke et al., 2004). Based on these considerations it is possible that a resonant network of connected FSIs in the neostriatum can selectively phase lock to the oscillatory component(s) of excitatory inputs and further, that the activity of these interneurons is partially responsible for the observed gamma activity in the LFP and the moderate phase locking of a subset of SPNs. In addition to $\mathrm{PV}^{+}$FSIs, $\mathrm{CR}^{+}$and $\mathrm{TH}^{+}$interneuron networks may also contribute, possibly with the three cell types playing distinct roles in the low $(\sim 50 \mathrm{~Hz})$ and high $(\sim 80 \mathrm{~Hz})$ frequency gamma oscillations observed in the striatum. The differential sensitivity of the two types of oscillations to psychostimulants (Berke, 2008, 2009) may also reflect the involvement of different GABAergic circuits.

With regard to the function of the diversity of GABAergic interneurons a comparison with the functional organization of interneurons in hippocampus and the neocortex may be instructive. This comparison 
is well motivated considering the shared developmental origin and in some respects striking similarity of interneurons in the striatum and in cortical structures (i.e., the neocortex and the hippocampus), including the pattern of expression of a similar complement of calcium binding proteins and neuropeptides as well as the similarity of the physiological properties and connectivity of striatal FSIs and basket cells in cortical areas mentioned above (Kawaguchi and Kubota, 1993). It is equally important to note however, that the 20 or more GABAergic interneurons identified in the CA1 field of the hippocampus greatly outnumber those currently recognized in the striatum and in most cases do not correspond to specific types of striatal interneurons. While it is likely that further investigation will reveal significantly more diversity among striatal interneurons than known today the homology of interneurons between the striatum and cortical structures will most likely be manifested as a common logic of cell type determination (and hence rules of classification) and not as a detailed correspondence of the majority of individual cell types.

These complexities not withstanding we believe that useful insights may be gained from certain emerging principles of the organization of hippocampal GABAergic interneurons and circuits. For a comprehensive discussion of these principles the reader is referred to the excellent recent review of Klausberger and Somogyi (2008), only the most relevant issues will be mentioned here. First, GABAergic inhibition of pyramidal neurons is highly organized, so that functionally distinct subcellular domains, including the axon initial segment, the soma, proximal and distal dendrites and even dendritic spines receive innervation from a unique but partially overlapping complement of GABAergic interneuron types. Conversely, individual types of interneurons display precise selectivity in innervating different postsynaptic subcellular domains. In the neostriatum, the observation of perisynaptic versus mostly dendritic localization of $\mathrm{PV}^{+}$and $\mathrm{NPY}^{+}$terminals respectively on SPNs (Kubota and Kawaguchi, 1993) suggests a similar specialization of interneurons.

Second several classes of interneurons have been discovered in the hippocampus that innervate primarily or exclusively other GABAergic interneurons. In principle the effect of these "higher order" neurons on the overall network activity may be significantly

\section{REFERENCES}

Abercrombie, E. D., and DeBoer, P. (1997). Substantia nigra D1 receptors and stimulation of striatal cholinergic interneurons by dopamine: a proposed circuit mechanism. J. Neurosci. 17, 8498-8505.

Adams, C. E., and Fisher, R. S. (1990). Sources of neostriatal cholecystokinin in the cat. J. Comp. Neurol. 992, 563-574.

Aoki, C., and Pickel, V. M. (1988). Neuropeptide Y-containing neurons in the rat striatum: ultrastructure and cellular relations with tyrosine hydroxylase- containing terminals and with astrocytes. Brain Res. 459, 205-225.

Bartos, M., Vida, I., and Jonas, P. (2007). Synaptic mechanisms of synchronized gamma oscillations in inhibitory interneuron networks. Nat. Rev. Neurosci. 8, 45-56.
Bennett, B. D., and Bolam, J. P. (1993). Characterization of calretinin-immunoreactive structures in the striatum of the rat. Brain Res. 609, 137-148.

Bennett, B. D., and Bolam, J. P. (1994a). Localisation of parvalbuminimmunoreactive structures in primate caudate-putamen. J. Comp. Neurol. 347, 340-356.

Bennett, B. D., and Bolam, J. P. (1994b). Synaptic input and output of parvalbumin-immunoreactive neurons in the neostriatum of the rat. Neuroscience 62, 707-719.

Berke, J. D. (2008). Uncoordinated firing rate changes of striatal fast-spiking interneurons during behavioral task performance. J. Neurosci. 28, 10075-10080.

Berke, J. D. (2009). Fast oscillations in cortical-striatal networks switch frequency following rewarding events

amplified through their control of powerful inhibitory circuits potentially resulting in a degree of influence that may not be readily predicted from their population size, the density of their axonal arborization, or the strength of their unitary synaptic connections. Therefore it will be interesting to examine if infrequent types of striatal interneurons, especially $\mathrm{TH}^{+}$interneurons provide significant inhibition of other interneurons. This possibility is supported by our preliminary optogenetic experiments showing synaptic inhibition of FSIs by TH interneurons (English et al., unpublished).

Finally, investigation of the firing activity of several identified types of interneurons in the hippocampus in relation to the three main oscillatory patterns of the hippocampus (theta rhythm, gamma oscillations, and high frequency ripples) revealed that there is no unique correspondence between individual cell types and inhibitory network functions. Instead, specific inhibitory functions (such as inhibition of distinct subcellular compartments, or inhibition associated with different oscillations) are provided cooperatively by multiple interneuron types and conversely, each interneuron type contributes to more then one (but not all) distinct inhibitory functions (Klausberger and Somogyi, 2008). This organization probably allows the fine tuning of each inhibitory function through complementing the unique properties, such as intrinsic firing properties, neuromodulation or use dependent plasticity of synaptic output, offered by individual types neurons. By analogy, one might expect a similar functional overlap between various neostriatal interneuron types with significant implications for future in vivo and in vitro investigation of the functioning of interneurons.

\section{ACKNOWLEDGMENTS}

We thank Dr. Elizabeth D. Abercrombie for generously allowing us the use of her microscopes and image acquisition. We thank Fulva Shah for 14 years of outstanding technical and administrative assistance, Harry Xenias for data shown in Figures $\mathbf{1}$ and $\mathbf{8}$ and Bengi Unal for helpful comments on the manuscript. Finally, we thank Leticia Maldonado for the reconstructions of the NPY neuron in Figure 3. Supported by NIH Grants NS034865 (James M. Tepper) and NS052370 (Tibor Koós) and Rutgers University.

and stimulant drugs. Eur. J. Neurosci. 30, 848-859.

Berke, J. D., Okatan, M., Skurski, J., and Eichenbaum,H.B. (2004). Oscillatory entrainment of striatal neurons in freely moving rats. Neuron 43, 883-896.

Bernard, V., Laribi, O., Levey, A. I., and Bloch, B. (1998). Subcellular redistribution of $\mathrm{m} 2$ muscarinic acetylcholine receptors in striatal interneurons in vivo after acute cholinergic stimulation. J. Neurosci. 18, 10207-10218.

Betarbet, R., Turner, R., Chockkan, V., DeLong, M. R., Allers, K. A., Walters, J., Levey, A. I., and Greenamyre, J. T. (1997). Dopaminergic neurons intrinsic to the primate striatum. J. Neurosci. 17, 6761-6768.

Bevan, M. D., Booth, P.A., Eaton, S. A., and Bolam, J. P. (1998). Selective innervation of neostriatal interneurons by a subclass of neuron in the globus pallidus of the rat. J. Neurosci. 18, 9438-9452.

Bolam, J. P., Clarke, D. J., Smith, A. D., and Somogyi, P. (1983). A type of aspiny neuron in the rat neostriatum accumulates $[3 \mathrm{H}]$ g-aminobutyric acid: combination of Golgi-staining, autoradiography, and electron microscopy. J. Comp. Neurol. 213, 121-134.

Bolam, J. P., Powell, J. F., Wu, J. Y., and Smith, A. D. (1985). Glutamate decarboxylase-immunoreactive structures in the rat neostriatum: a correlated light and electron microscopic study including a combination of Golgi impregnation with immunocytochemistry. J. Comp. Neurol. 237, 1-20.

Bracci, E., Centonze, D., Bernardi, G., and Calabresi, P. (2002). Dopamine excites fast-spiking interneurons in the striatum. J. Neurophysiol. 87, 2190-2194. 
Bracci, E., Centonze, D., Bernardi, G., and Calabresi,P. (2003).Voltage-dependent membrane potential oscillations of rat striatal fast-spiking interneurons. J. Physiol. 549, 121-130.

Busceti, C. L., Biagioni, F., Mastroiacovo, F., Bucci, D., Lenzi, P., Pasquali, L., Trabucco, A., Nicoletti, F., and Fornai, F. (2008). High number of striatal dopaminergic neurons during early postnatal development: correlation analysis with dopaminergic fibers. J. Neural. Transm. 115, 1375-1383.

Centonze, D., Bracci, E., Pisani, A., Gubellini, P., Bernardi, G., and Calabresi, P. (2002). Activation of dopamine D1-like receptors excites LTS interneurons of the striatum. Eur. J. Neurosci. 15, 2049-2052.

Centonze, D., Grande, C., Usiello, A., Gubellini, P., Erbs, E., Martin, A. B., Pisani, A., Tognazzi, N., Bernardi, G., Moratalla, R., Borrelli, E., and Calabresi, P. (2003). Receptor subtypes involved in the presynaptic and postsynaptic actions of dopamine on striatal interneurons. $J$. Neurosci. 23, 6245-6254.

Chang, H. T., and Kita, H. (1992). Interneurons in the rat striatum: relationships between parvalbumin neurons and cholinergic neurons. Brain Res. 574, 307-311.

Chesselet, M. F., and Graybiel, A. M. (1986). Striatal neurons expressing somatostatin-like immunoreactivity: evidence for a peptidergic interneuronal system in the cat. Neuroscience $17,547-571$.

Chesselet, M. F., and Robbins, E. (1989). Characterization of striatal neurons expressing high levels of glutamic acid decarboxylase messenger RNA. Brain Res. 492, 237-244.

Cossette, M., Levesque, D., and Parent, A. (2005). Neurochemical characterization of dopaminergic neurons in human striatum. Parkinsonism Relat. Disord. 11, 277-286.

Cossette, M., Parent, A., and Levesque, D. (2004). Tyrosine hydroxylase-positive neurons intrinsic to the human striatum express the transcription factor Nurr1. Eur. J. Neurosci. 20, 2089-2095.

Cowan, R. L., Wilson, C. J., Emson, P. C., and Heizmann, C. W. (1990). Parvalbumin-containing GABAergic interneurons in the rat neostriatum. J. Comp. Neurol. 302, 197-205.

Czubayko, U., and Plenz, D. (2002). Fast synaptic transmission between striatal spiny projection neurons. Proc. Natl. Acad. Sci. U.S.A. 99, 15764-15769.

Darmopil, S., Muneton-Gomez, V. C., de Ceballos, M. L., Bernson, M., and Moratalla, R. (2008). Tyrosine hydroxylase cells appearing in the mouse striatum after dopamine denervation are likely to be projection neurones regulated by L-DOPA. Eur. J. Neurosci. $27,580-592$.

DiFiglia, M., and Aronin, N. (1982). Ultrastructural features of immunoreactive somatostatin neurons in the rat caudate nucleus. J. Neurosci. 2, 1267-1274.

Dubach, M., Schmidt, R., Kunkel, D., Bowden, D. M., Martin, R., and German, D. C. (1987). Primate neostriatal neurons containing tyrosine hydroxylase: immunohistochemical evidence. Neurosci. Lett. 75, 205-210.

Figueredo-Cardenas, G., Morello, M., Sancesario, G., Bernardi, G., and Reiner, A. (1996). Colocalization of somatostatin, neuropeptide $\mathrm{Y}$, neuronal nitric oxide synthase and NADPHdiaphorase in striatal interneurons in rats. Brain Res. 735, 317-324.

Freiman, I., Anton, A., Monyer, H., Urbanski, M. J., and Szabo, B. (2006). Analysis of the effects of cannabinoids on identified synaptic connections in the caudate-putamen by paired recordings in transgenic mice. J. Physiol. 575, 789-806.

Freund, T. F., and Buzsáki, G. (1996). Interneurons of the hippocampus. Hippocampus 6, 347-470.

Fujiyama, F., and Masuko, S. (1996). Association of dopaminergic terminals and neurons releasing nitric oxide in the rat striatum: an electron microscopic study using NADPH-diaphorase histochemistry and tyrosine hydroxylase immunohistochemistry. Brain Res. Bull. 40, 121-127.

Gage, G. J., Stoetzner, C. R., Wiltschko, A. B., and Berke, J. D. (2010). Selective activation of striatal fast-spiking interneurons during choice execution. Neuron 67, 466-479.

Galarreta, M., and Hestrin, S. (1999). A network of fast-spiking cells in the neocortex connected by electrical synapses. Nature 402, 72-75.

Galarreta, M., and Hestrin, S. (2001). Spike transmission and synchrony detection in networks of GABAergic interneurons. Science 292, 2295-2299.

Galarreta, M., and Hestrin, S. (2002). Electrical and chemical synapses among parvalbumin fast-spiking GABAergic interneurons in adult mouse neocortex. Proc. Natl. Acad. Sci. U.S.A. 99, 12438-12443.

Gerfen, C. R., Baimbridge, K. G., and Miller, J. J. (1985). The neostriatal mosaic: compartmental distribution of calcium-binding protein and parvalbumin in the basal ganglia of the rat and monkey. Proc. Natl. Acad. Sci. U.S.A. 82, 8780-8784.

Gerfen, C. R., and Wilson, C. J. (1996). "The basal ganglia," in Handbook of Chemical Neuroanatomy, 12th Edn, eds L. W. Swanson, A. Bjorklund, and T. Hokfelt (Amsterdam: Elsevier Science), 371-468.

Gilles, C., Lotstra, F., and Vanderhaeghen, J.-J. (1983). CCK nerve terminals in the rat striatal and limbic areas originate partly in the brain stem and partly in telencephalic structures. Life Sci. 32, 1683-1690.

Gittis, A. H., Nelson, A. B., Thwin, M. T., Palop, J. J., and Kreitzer, A. C. (2010). Distinct roles of GABAergic interneurons in the regulation of striatal output pathways. J. Neurosci. 30, 2223-2234.

Gong, S., Zheng, C., Doughty, M. L., Losos, K., Didkovsky, N., Schambra, U. B., Nowak, N. J., Joyner. A., Leblanc, G., Hatten, M. E., and Heintz, N. (2003). A gene expression atlas of the central nervous system based on bacterial artificial chromosomes. Nature 425, 917-925.

Graveland, G. A., and DiFiglia, M. (1985). The frequency and distribution of medium-sized neurons with indented nuclei in the primate and rodent neostriatum. Brain Res. 327, 307-311

Grofova, I. (1979). Types of striatonigral neurons labeled by retrograde transport of horseradish peroxidase. Appl. Neurophysiol. 42, 25-28.

Gustafson, N., Gireesh-Dharmaraj, E., Czubayko, U., Blackwell, K. T., and Plenz, D. (2006). A comparative voltage and current-clamp analysis of feedback and feedforward synaptic transmission in the striatal microcircuit in vitro. J. Neurophysiol. 95, 737-752.

Hökfelt, T., Herrera-Marschitz, M. Seroogy, K., Ju, G., Staines, W. A., Holets, V., Schalling, M., Ungerstedt, U., Post, C., and Rehfeld, J. F. (1988). Immunohistochemical studies on cholecystokinin (CCK)immunoreactive neurons in the rat using sequence specific antisera and with special reference to the caudate nucleus and primary sensory neurons. J. Chem. Neuroanat. 1, 11-51.

Hope, B. T., Michael, G. J., Knigge, K. M., and Vincent, S. R. (1991). Neuronal NADPH diaphorase is a nitric oxide synthase. Proc. Natl. Acad. Sci. U.S.A. 88, 2811-2814.

Huot, P., Levesque, M., and Parent, A. (2007). The fate of striatal dopaminergic neurons in Parkinson's disease and Huntington's chorea. Brain 130, 222-232.

Huot, P., and Parent, A. (2007). Dopaminergic neurons intrinsic to the striatum. J. Neurochem. 101, 1441-1447.

Ibáñez-Sandoval, O., Tecuapetla, F., Unal, B., Shah, F., Koós, T., and Tepper, J. M. (2010). Electrophysiological and morphological characteristics and synaptic connectivity of tyrosine hydroxylase- expressing neurons in adult mouse striatum. J. Neurosci. 30, 6999-7016.

Jollivet, C., Montero-Menei, C. N., Venier-Julienne, M. C., Sapin, A., Benoit, J. P., and Menei, P. (2004). Striatal tyrosine hydroxylase immunoreactive neurons are induced by L-dihydroxyphenylalanine and nerve growth factor treatment in 6-hydroxydopamine lesioned rats. Neurosci. Lett. 362, 79-82.

Kalenscher, T., Lansink, C. S., Lankelma, J. V., and Pennartz, C.M. (2010). Rewardassociated gamma oscillations in ventral striatum are regionally differentiated and modulate local firing activity. $J$. Neurophysiol. 103, 1658-1672.

Kawaguchi, Y. (1993). Physiological, morphological, and histochemical characterization of three classes of interneurons in rat neostriatum. $J$. Neurosci. 13, 4908-4923.

Kawaguchi, Y. (1997). Neostriatal cell subtypes and their functional roles. Neurosci. Res. 27, 1-8.

Kawaguchi, Y., and Kubota, Y. (1993). Correlation of physiological subgroupings of nonpyramidal cells with parvalbumin- and calbindinD28kimmunoreactive neurons in layer $\mathrm{V}$ of rat frontal cortex. J. Neurophysiol. 70, 387-396.

Kawaguchi, Y., Wilson, C. J., Augood, S. J., and Emson, P. C. (1995). Striatal interneurones: chemical, physiological and morphological characterization. Trends Neurosci. 18, 527-535.

Kimura, H., McGeer, P. L., Peng, F., and McGeer, E. G. (1980). Choline acetyltransferase-containing neurons in rodent brain demonstrated by immunohistochemistry. Science 208, 1057-1059.

Kita, H. (1993). GABAergic circuits of the striatum. Prog. Brain Res. 99, 51-72.

Kita, H., Kosaka, T., and Heizmann, C.W. (1990). Parvalbumin-immunoreactive neurons in the rat neostriatum: a light and electron microscopic study. Brain Res. 536, 1-15.

Klausberger, T., and Somogyi, P. (2008). Neuronal diversity and temporal dynamics: the unity of hippocampal circuit operations. Science 321, 53-57.

Kölliker, A. (1896). Handbuch der Geweleher des Menschen, Vol 2, Nervensystem. Leipzig: Engelmann.

Koós, T., and Tepper, J. M. (1999). Inhibitory control of neostriatal projection neurons by GABAergic interneurons. Nat. Neurosci. 2, 467-472.

Koós, T., and Tepper, J. M. (2002). Dual cholinergic control of fast-spiking interneurons in the neostriatum. J. Neurosci. 22, 529-535.

Koós, T., Tepper, J. M., and Wilson, C. J. (2004). Comparison of IPSCs evoked 
by spiny and fast-spiking neurons in the neostriatum. J. Neurosci. 24, 7916-7922.

Kubota, Y., Inagaki, S., Kito, S., Shimada, S., Okayama, T., Hatanaka, H., Pelletier, G., Takagi,H., and Tohyama, M. (1988). Neuropeptide Y-immunoreactive neurons receive synaptic inputs from dopaminergic axon terminals in the rat neostriatum. Brain Res. 458, 389-393.

Kubota, Y., Inagaki, S., Kito, S., and Wu, J. Y.(1987). Dopaminergic axons directly make synapses with GABAergic neurons in the rat neostriatum. Brain Res. 406, 147-156.

Kubota, Y., and Kawaguchi, Y. (1993). Spatial distributions of chemically identified intrinsic neurons in relation to patch and matrix compartments of rat neostriatum. J. Comp. Neurol. 332, 499-513.

Kubota, Y., and Kawaguchi, Y. (1994). Three classes of GABAergic interneurons in neocortex and neostriatum. Jpn. J. Physiol. 44, S145-S148.

Kubota, Y., and Kawaguchi, Y. (1995). Three distinct classes of GABAergic interneurons in neocortex and neostriatum. Riken Rev. 9, 15-16.

Kubota, Y., and Kawaguchi, Y. (2000). Dependence of GABAergic synaptic areas on the interneuron type and target size. J. Neurosci. 20, 375-386.

Kubota, Y., Mikawa, S., and Kawaguchi, Y. (1993). Neostriatal GABAergic interneurones contain NOS, calretinin or parvalbumin. Neuroreport 5, 205-208.

Laurent, G. (2002). Olfatory network dynamics and the coding of multidimensional signals. Nat. Rev. Neurosci. 3, 884-895.

Lenz, S., Perney, T.M., Qin, Y., Robbins, E., and Chesselet, M.F.(1994). GABAergic interneurons of the striatum express the shaw-like potassium channel Kv3.1. Synapse 18, 55-66.

Lopez-Huerta, V. G., Tecuapetla, F., Guzman,J.N., Bargas,J., and Galarraga, E. (2008). Presynaptic modulation by somatostatin in the neostriatum. Neurochem. Res. 33, 1452-1458.

Lopez-Real, A., Rodriguez-Pallares, J., Guerra,M.J., and Labandeira-Garcia,J. L. (2003). Localization and functional significance of striatal neurons immunoreactive to aromatic $\mathrm{L}$-amino acid decarboxylase or tyrosine hydroxylase in rat Parkinsonian models. Brain Res. 969, 135-146.

Luk, K. C., and Sadikot, A. F. (2001). GABA promotes survival but not proliferation of parvalbumin-immunoreactive interneurons in rodent neostriatum: an in vivo study with stereology. Neuroscience 104, 93-103.

Mallet, N., Le Moine, C., Charpier, S., and Gonon, F. (2005). Feedforward inhibition of projection neurons by fast-spiking GABA interneurons in the rat striatum in vivo. J. Neurosci. 25, 3857-3869.

Mao, L., Lau, Y.S., Petroske, E., and Wang, J. Q. (2001). Profound astrogenesis in the striatum of adult mice following nigrostriatal dopaminergic lesion by repeated MPTP administration. Brain. Res. Dev. Brain Res. 131, 57-65.

Marin, O.,Anderson,S.A., and Rubenstein, J. L. (2000). Origin and molecular specification of striatal interneurons. J. Neurosci. 20, 6063-6076.

Mazloom, M., and Smith, Y. (2006). Synaptic microcircuitry of tyrosine hydroxylase-containing neurons and terminals in the striatum of 1-methyl4-phenyl-1,2,3,6-tetrahydropyridinetreated monkeys. J. Comp. Neurol. 495, 453-469.

Mehler, W. R. (1981). The basal gangliacirca 1982. Appl. Neurophysiol. 44, 261-290.

Meredith, G. E., Farrell, T., Kellaghan, P., Tan, Y., Zahm, D. S., and Totterdell, S. (1999). Immunocytochemical characterization of catecholaminergic neurons in the rat striatum following dopamine-depleting lesions. Eur. J. Neurosci. 11, 3585-3596.

Mura, A., Jackson, D., Manley, M. S., Young, S. J., and Groves, P. M. (1995). Aromatic L-amino acid decarboxylase immunoreactive cells in the rat striatum: a possible site for the conversion of exogenous L-DOPA to dopamine. Brain Res. 704, 51-60.

Mura, A., Linder, J. C., Young, S. J., and Groves, P. M. (2000). Striatal cells containing aromatic L-amino acid decarboxylase: an immunohistochemical comparison with other classes of striatal neurons. Neuroscience 98, 501-511.

Pais, I., Hormuzdi, S. G., Monyer, H., Traub, R. D., Wood, I. C., Buhl, E. H., Whittington, M. A., and LeBeau, F. E. (2003). Sharp wave-like activity in the hippocampus in vitro in mice lacking the gap junction protein connexin 36. J. Neurophysiol. 89, 2046-2054.

Palfi, S., Leventhal, L., Chu, Y., Ma, S. Y., Emborg, M., Bakay, R., Deglon, N., Hantraye, P., Aebischer, P., and Kordower, J. H. (2002). Lentivirally delivered glial cell line-derived neurotrophic factor increases the number of striatal dopaminergic neurons in primate models of nigrostriatal degeneration. J. Neurosci. 22, 4942-4954.

Parent, A. (1986). Comparative Neurobiology of the Basal Ganglia. New York: John Wiley \& Sons.

Parthasarathy, H. B., and Graybiel, A. M. (1997). Cortically driven immediate-early gene expression reflects modular influence of sensorimotor cortex on identified striatal neurons in the squirrel monkey.J. Neurosci. 17, 2477-2491.

Partridge, J. G., Janssen, M. J., Chou, D. Y., Abe, K., Zukowska, Z., and Vicini, S. (2009). Excitatory and inhibitory synapses in neuropeptide Y-expressing striatal interneurons. J. Neurophysiol. 102, 3038-3045.

Planert, H., Szydlowski, S. N., Hjorth, J. J., Grillner, S., and Silberberg, G. (2010). Dynamics of synaptic transmission between fast-spiking interneurons and striatal projection neurons of the direct and indirect pathways. $J$. Neurosci. 30, 3499-3507.

Plenz, D., and Kitai, S. T. (1998). Up and down states in striatal medium spiny neurons simultaneously recorded with spontaneous activity in fast-spiking interneurons studied in cortexstriatum-substantia nigra organotypic cultures. J. Neurosci. 18, 266-283.

Plotkin, J. L., Wu, N., Chesselet, M. F., and Levine, M. S. (2005). Functional and molecular development of striatal fastspiking GABAergic interneurons and their cortical inputs. Eur. J. Neurosci. 22, 1097-1108.

Popescu, A. T., Popa, D., and Paré, D. (2009). Coherent gamma oscillations couple the amygdala and striatum during learning. Nat. Neurosci. 12, 801-807.

Porrit, M. J., Kingsbury, A. E., Hughes, A. J., and Howells, D. W. (2006). Striatal dopaminergic neurons are lost with Parkinson's disease progression. Mov. Disord. 21, 2208-2211.

Prensa, L., Giménez-Amaya, J. M., and Parent, A. (1998). Morphological features of neurons containing calcium-binding proteins in the human striatum. J. Comp. Neurol. 390, 552-563.

Ramanathan, S., Hanley, J. J., Deniau, J. M., and Bolam, J. P. (2002). Synaptic convergence of motor and somatosensory cortical afferents onto GABAergic interneurons in the rat striatum. $J$. Neurosci. 22, 8158-8169.

Ramon y Cajal, S. (1911). Histologie du Systeme Nerveux de l'Homme et des Vertébrés. Paris: Maloine.

Rudy, B., and McBain, C. J. (2001). Kv3 channels: voltage-gated $\mathrm{K}+$ channels designed for high-frequency repetitive firing. Trends Neurosci. 24, 517-526.

Rymar, V.V., Sasseville, R., Luk, K. C., and Sadikot, A. F. (2004). Neurogenesis and stereological morphometry of calretinin-immunoreactive GABAergic interneurons of the neostriatum. J. Comp. Neurol. 469, 325-339.

San Sebastián, W., Guillén, J., Manrique, M., Belzunegui, S., Ciordia, E., IzalAzcárate, A., Garrido-Gil, P., VázquezClaverie, M., and Luquin, M. R. (2007).
Modification of the number and phenotype of striatal dopaminergic cells by carotid body graft. Brain 130, 1306-1316.

Schiffmann, S. N., Mailleux, P., Przedborski, S., Halleux, P., Lotsra, F., and Vanderhaeghen, J.-J. (1989). Cholecystokinin distribution in the human striatum and related subcortical structures. Neurochem. Int. 14, 167-173.

Schlosser, B., Klausa, G., Prime, G., and Ten Bruggencate, G. (1999). Postnatal development of calretinin- and parvalbumin-positive interneurons in the rat neostriatum: an immunohistochemical study. J. Comp. Neurol. 405, 185-198.

Sullivan, M.A., Chen, H., and Morikawa, H. (2008). Recurrent inhibitory network among striatal cholinergic interneurons. J. Neurosci. 28, 8682-8690.

Takagi, H., Somogyi, P., and Smith, A. D. (1984a). Aspiny neurons and their local axons in the neostriatum of the rat: a correlated light and electron microscopic study of Golgiimpregnated material. J. Neurocytol. 13, 239-265.

Takagi, H., Mizuta, H., Matsuda, T., Inagaki, S., Tateishi, K., and Hamaoka, T. (1984b). The occurrence of cholecystokinin-like immunoreactive neurons in the rat neostriatum: light and electron microscopic analysis. Brain Res. 309, 346-349.

Tande, D., Hoglinger, G., Debeir, T., Freundlieb, N., Hirsch, E. C., and Francois, C. (2006). New striatal dopamine neurons in MPTP-treated macaques result from a phenotypic shift and not neurogenesis. Brain 129, 1194-1200.

Tashiro, Y., Kaneko, T., Sugimoto, T., Nagatsu, I., Kikuchi, H., and Mizuno, N. (1989a). Striatal neurons with aromatic L-amino acid decarboxylase-like immunoreactivity in the rat. Neurosci. Lett. 100, 29-34.

Tashiro, Y., Sugimoto, T., Hattori, T., Uemura, Y., Nagatsu, I., Kikuchi, H., and Mizuno, N. (1989b). Tyrosine hydroxylase-like immunoreactive neurons in the striatum of the rat. Neurosci. Lett. 97, 6-10.

Taverna, S., Canciani, B., and Pennartz, C. M. (2007). Membrane properties and synaptic connectivity of fast-spiking interneurons in rat ventral striatum. Brain Res. 1152, 49-56.

Taverna, S., van Dongen, Y. C., Groenewegen, H. J., and Pennartz, C. M. (2004). Direct physiological evidence for synaptic connectivity between medium-sized spiny neurons in rat nucleus accumbens in situ. $J$. Neurophysiol. 91, 1111-1121.

Tecuapetla, F., Koos, T., Tepper, J. M., Kabbani, N., and Yeckel, M. F. (2009). 
Differential dopaminergic modulation of neostriatal synaptic connections of striatopallidal axon collaterals. J. Neurosci. 29, 8977-8990.

Tepper,J.M. (2010). “GABAergic interneurons of the striatum," in Handbook of Basal Ganglia Structure and Function, eds.H.Steiner and K. Tseng (Amsterdam: Academic Press), 151-166.

Tepper, J. M., and Bolam, J. P. (2004). Functional diversity and specificity of neostriatal interneurons. Curr. Opin. Neurobiol. 14, 685-692.

Tepper, J. M., Koos, T., and Wilson, C. J. (2004). GABAergic microcircuits in the neostriatum. Trends Neurosci. 27, 662-669.

Tepper, J. M., Wilson, C. J., and Koos, T. (2008). Feedforward and feedback inhibition in neostriatal GABAergic spiny neurons. Brain Res. Rev. 58, 272-281.

Theirault, E., and Lamdis, D. M. (1987). Morphology of striatal neurons containing VIP-like immunoreactivity. J. Comp. Neurol. 256, 1-13.

Traub, R.D.,Jefferys, J.G., and Whittington, M. A. (1999). Functionally relevant and functionally disruptive (epileptic) synchronized oscillations in brain slices. Adv. Neurol. 79, 709-724.

Tsai, H. C., Zhang, F., Adamantidis, A., Stuber, G. D., Bonci, A., de Lecea, L., and Deisseroth, K. (2009). Phasic firing in dopaminergic neurons is sufficient for behavioral conditioning. Science 324, 1080-1084.

Tunstall, M. J., Oorschot, D. E., Kean, A., and Wickens, J. R. (2002). Inhibitory interactions between spiny projection neurons in the rat striatum. $J$. Neurophysiol. 88, 1263-1269.

van der Meer, M. A., and Redish, A. D. (2009). Low and high gamma oscillations in rat ventral striatum have distinct relationships to behavior, reward, and spiking activity on a learned spatial decision task. Front. Integr. Neurosci. 3:9. doi: 10.3389/ neuro.07.009.2009.

Vincent, S. R., and Johansson, O. (1983). Striatal neurons containing both somatostatin- and avian pancreatic polypeptide (APP)-like immunoreactivities and NADPH-diaphorase activity: a light and electron microscopic study. J. Comp. Neurol. 217, 264-270.

Vincent, S. R., Johansson, O., Hokfelt, T., Skirboll, L., Elde, R. P., Terenius, L., Kimmel, J., and Goldstein, M. (1983). NADPH-Diaphorase: a selective histochemical marker for striatal neurons containing both somatostatin- and avian pancreatic polypeptide (APP)like immunoreactivities. J. Comp. Neurol. 217, 252-263.

Vogt, C., and Vogt, O. (1920). Zur Lehre der Erkranungen des striären Systems. J. Psychol. Neurol. (Leipzig) 25, 627-846.

Vuillet, J., Dimova, R., Nieoullon, A., and Kerkerian-Le Goff, L. (1992). Ultrastructural relationships between choline acetyltransferase- and neuropeptide Y-containing neurons in the rat striatum. Neuroscience 46, 351-360.

Vuillet, J., Kerkerian, L., Salin, P., and Nieoullon,A. (1989a). Ultrastructural features of NPY-containing neurons in the rat striatum. Brain Res. 477, 241-251.

Vuillet, J., Kerkerian, L., Kachidian, P. Bosler, O., and Nieoullon, A. (1989b). Ultrastructural correlates of functional relationships between nigral dopaminergic or cortical afferent fibers and neuropeptide Y-containing neurons in the rat striatum. Neurosci. Lett. 100, 99-104.

Wilson, C. J., and Groves, P. M. (1980). Fine structure and synaptic connections of the common spiny neuron of the rat neostriatum: a study employing intracellular inject of horseradish peroxidase. J. Comp. Neurol. 194, 599-615.

Wilson, C. J., and Kawaguchi, Y. (1996). The origins of two-state spontaneous membrane potential fluctuations of neostriatal spiny neurons. J. Neurosci. 16, 2397-2410.

Wiltschko, A. B., Pettibone, J. R., and Berke, J. D. (2010). Opposite effects of stimulant and antipsychotic drugs on striatal fast-spiking interneurons. Neuropsychopharmacology 35, 1261-1270.

Wu, Y., and Parent, A. (2000). Striatal interneurons expressing calretinin, parvalbumin or NADPH- diaphorase: a comparative study in the rat, monkey and human. Brain Res. 863 , 182-191.

Yan, Z., You, Y., and Levison, S. W. (2008). Neonatal hypoxic/ischemic brain injury induces production of calretinin-expressing interneurons in the striatum. J. Comp. Neurol. 511, 19-33.

Záborszky, L., Alheid, G. F., Beinfeld, M. C., Eiden, L. E., Heimer, L., and Palkovits, M. (1985). Cholecystokinin innervation of the ventral striatum: a morphological and radioimmunological study. Neuroscience 14, 427-453.

Zhou, F. M., Wilson, C. J., and Dani, J. A. (2002). Cholinergic interneuron characteristics and nicotinic properties in the striatum. J. Neurobiol. 53, 590-605.

Conflict of Interest Statement: The authors declare that the research was conducted in the absence of any commercial or financial relationships that could be construed as a potential conflict of interest.

Received: 22 November 2010; accepted: 10 December 2010; published online: 29 December 2010.

Citation: Tepper JM, Tecuapetla F, Koós T and Ibáñez-Sandoval O (2010) Heterogeneity and diversity of striatal GABAergicinterneurons. Front. Neuroanat. 4:150. doi: 10.3389/fnana.2010.00150 Copyright (C) 2010. Tepper, Tecuapetla, Koós and Ibáñez-Sandoval. This is an open-access article subject to an exclusive license agreement between the authors and the Frontiers Research Foundation, which permits unrestricted use, distribution, and reproduction in any medium, provided the original authors and source are credited. 